\title{
Chronic cigarette smoke exposure induces systemic hypoxia that drives intestinal dysfunction
}

\author{
Michael Fricker, ${ }^{1,2}$ Bridie J. Goggins, ${ }^{2}$ Sean Mateer, ${ }^{2}$ Bernadette Jones, ${ }^{1,2}$ Richard Y. Kim, ${ }^{1,2}$ \\ Shaan L. Gellatly, ${ }^{1,2}$ Andrew G. Jarnicki, ${ }^{1,2}$ Nicholas Powell, ${ }^{3}$ Brian G. Oliver, ${ }^{4,5}$ \\ Graham Radford-Smith, ${ }^{6,7}$ Nicholas J. Talley, ${ }^{8}$ Marjorie M. Walker, ${ }^{8}$ Simon Keely, ${ }^{2,8}$ \\ and Philip M. Hansbro ${ }^{1,2,8}$ \\ 1Priority research Centre for Healthy Lungs, University of Newcastle and ${ }^{2}$ Hunter Medical Research Institute, Newcastle, \\ New South Wales, Australia. 'Faculty of Translational Medicine, Guy's and St. Thomas' and King's College London \\ Comprehensive Biomedical Research Centre, Great Maze Pond, London, United Kingdom. ${ }^{4}$ Woolcock Institute of Medical \\ Research, The University of Sydney, Sydney, New South Wales, Australia. ${ }^{5}$ School of Life Sciences, The University of \\ Technology, Sydney, New South Wales, Australia. ${ }^{6}$ Royal Brisbane and Women's Hospital, Brisbane, School of Medicine, \\ University of Queensland, and ${ }^{7}$ QIMR Berghofer Medical Research Institute, Brisbane, Queensland, Australia. ${ }^{8}$ Priority \\ Research Centre for Digestive Health and Neurogastroenterology, University of Newcastle, New South Wales, Australia.
}

Crohn's disease (CD) is a chronic inflammatory disease of the gastrointestinal tract (CIT). Cigarette smoke (CS) exposure and chronic obstructive pulmonary disease (COPD) are risk factors for CD, although the mechanisms involved are poorly understood. We employed a mouse model of CSinduced experimental COPD and clinical studies to examine these mechanisms. Concurrent with the development of pulmonary pathology and impaired gas exchange, CS-exposed mice developed CD-associated pathology in the colon and ileum, including gut mucosal tissue hypoxia, HIF-2 stabilization, inflammation, increased microvasculature, epithelial cell turnover, and decreased intestinal barrier function. Subsequent smoking cessation reduced GIT pathology, particularly in the ileum. Dimethyloxaloylglycine, a pan-prolyl hydroxylase inhibitor, ameliorated CS-induced GIT pathology independently of pulmonary pathology. Prior smoke exposure exacerbated intestinal pathology in 2,4,6-trinitrobenzenesulfonic acid-induced (TNBS-induced) colitis. Circulating vascular endothelial growth factor, a marker of systemic hypoxia, correlated with CS exposure and CD in mice and humans. Increased mucosal vascularisation was evident in ileum biopsies from CD patients who smoke compared with nonsmokers, supporting our preclinical data. We provide strong evidence that chronic CS exposure and, for the first time to our knowledge, associated impaired gas exchange cause systemic and intestinal ischemia, driving angiogenesis and GIT epithelial barrier dysfunction, resulting in increased risk and severity of CD.

Authorship note: SK and PMH contributed equally to this work.

Conflict of interest: The authors have declared that no conflict of interest exists.

Submitted: March 15, 2017 Accepted: January 10, 2018 Published: February 8, 2018

Reference information: JCI Insight. 2018;3(3):e94040. https://doi.org/10.1172/jci. insight. 94040.

\section{Introduction}

Chronic obstructive pulmonary disease (COPD) is the third leading cause of mortality and morbidity in the world, and its incidence is increasing (1). COPD describes a range of pulmonary pathologies including chronic bronchitis, small airway remodeling, and emphysema, which - in combination - result in reduced gas exchange and lung function $(2,3)$. Inhalation of noxious particulates - in particular cigarette smoke (CS), but also wood smoke, cooking smoke, and air pollution - are major risk factors for the development of COPD. Crohn's disease (CD), one of the 2 predominant forms of inflammatory bowel disease (IBD), is estimated to affect $0.5 \%$ of the population in Western societies, and its prevalence is increasing globally $(4,5) . \mathrm{CD}$ is characterized by chronic mucosal inflammation of the gastrointestinal tract (GIT) and compromised intestinal barrier function that gradually become more severe over time $(6,7)$. The etiology of $\mathrm{CD}$ is incompletely understood, but genetic, environmental, and disease risk factors play an important role.

CS exposure is also a major environmental risk factor for $\mathrm{CD}$, and the subsequent development of COPD further increases the risk of CD. Smokers and COPD sufferers have 2 and 2.72 times greater risk 
A

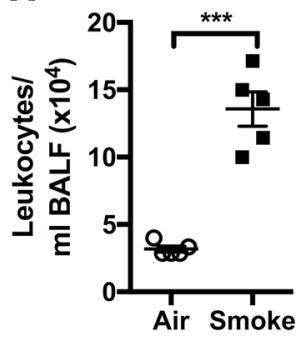

B

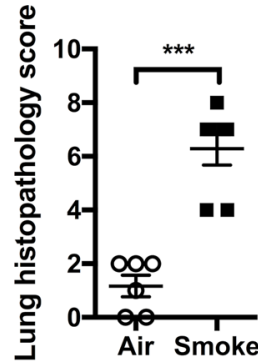

C

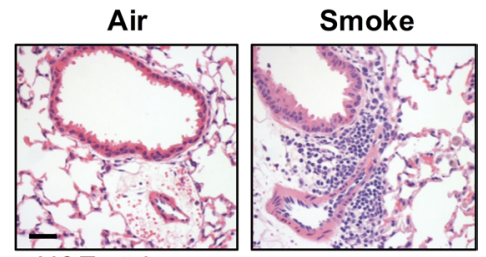

H\&E stain

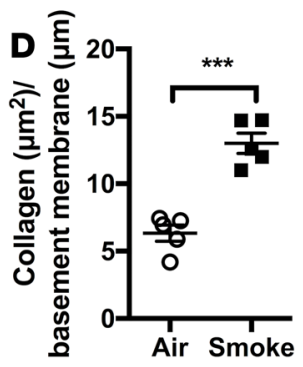

$\mathbf{E}$
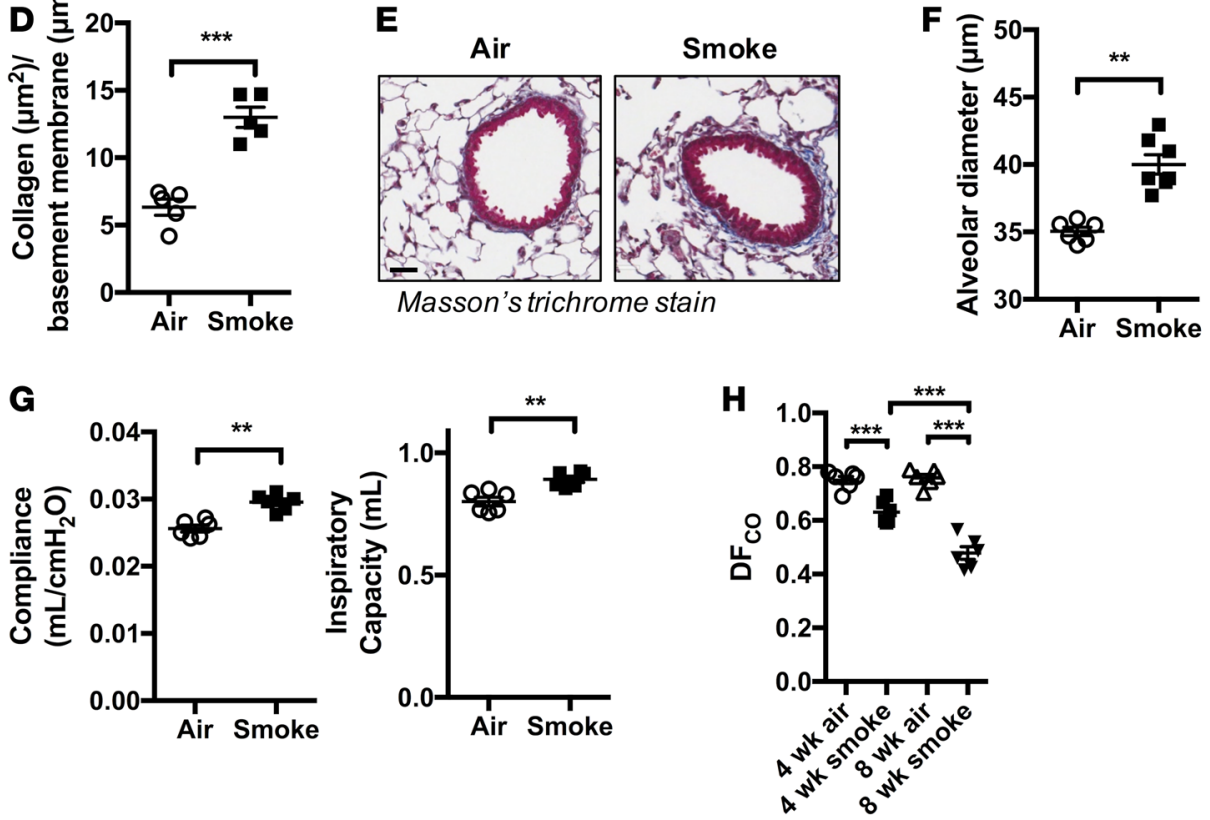

Figure 1. Chronic CS exposure induces experimental COPD and impairs gas exchange in the lung. Mice were exposed through the nose only to CS for 8 weeks to induce experimental COPD. (A) Bronchoalveolar lavage was performed and total airway leukocytes enumerated $(n=5)$. (B) Formalin fixed lung tissue was H\&E stained and evaluated by histopathological scoring for the presence of inflammatory cells in peribronchial, perivascular, and alveolar regions $(n=6)$. (C) Representative H\&E-stained tissue sections showing increased immune cell accumulation in peribronchial and perivascular areas of lungs of CS-exposed mice (scale bar: $50 \mu \mathrm{M}$ ). (D) Quantification of collagen deposition around small airways (perimeter $\leq 1,000 \mu \mathrm{M}$ ) indicating airway remodeling ( $n$ = 5). (E) Representative images of lung sections stained with Masson's trichrome used for airway collagen quantification (scale bar: $50 \mu M$ ). (F) CS-induced emphysema-like alveolar enlargement $(n=6-7)$. (C) Impaired lung function in terms of increased lung compliance and inspiratory capacity in experimental COPD $(n=6-7)$. (H) Mice were exposed to CS for 4 or 8 weeks to drive the development (4 weeks) and establishment (8 weeks) of experimental COPD, with reduced $\mathrm{DF}_{\mathrm{CO}}$ demonstrating impaired gas exchange in experimental COPD $(n=6)$. ${ }^{*} P \leq 0.01,{ }^{* *} P \leq 0.001$ air vs. smoke. Student's unpaired 2 -tailed $t$ test used for comparisons of 2 groups, 1-way ANOVA with Tukey's post-hoc was used whenever more than 2 experimental groups were compared.

for $\mathrm{CD}$, respectively $(8,9)$, and CS is associated with relapse and increased disease severity in $\mathrm{CD}$ patients (10). The GIT is increasingly recognized as a major site of extrapulmonary dysfunction that may be relevant to COPD patients $(6,11)$.

The mechanistic links between CS, COPD, and CD are poorly understood (6, 7). Several mechanisms have been proposed, including the effects of CS components such as nicotine on barrier and endothelial function, oxidative stress, smooth muscle contractility, and microbiome changes; however, to date, experimental data has not yet definitively supported these hypotheses $(9,11,12)$. Recently, it was reported that COPD patients exhibit increased intestinal barrier permeability associated with reduced lung function, both during rest and following mild physical exertion (13).

To better understand mechanisms of increased susceptibility to CD associated with CS and COPD, we employed a pathophysiologically relevant mouse model of CS exposure and experimental COPD. We have previously demonstrated that exposure of mice to mainstream CS for 8 weeks induces chronic airway inflammation, leading to pathology and altered lung function reminiscent of $\operatorname{COPD}(2,14-20)$.

Here, we demonstrate that chronic (8-week) CS exposure is associated with the induction of impaired lung gas exchange capacity and systemic hypoxia using our preclinical model and clinical samples. 

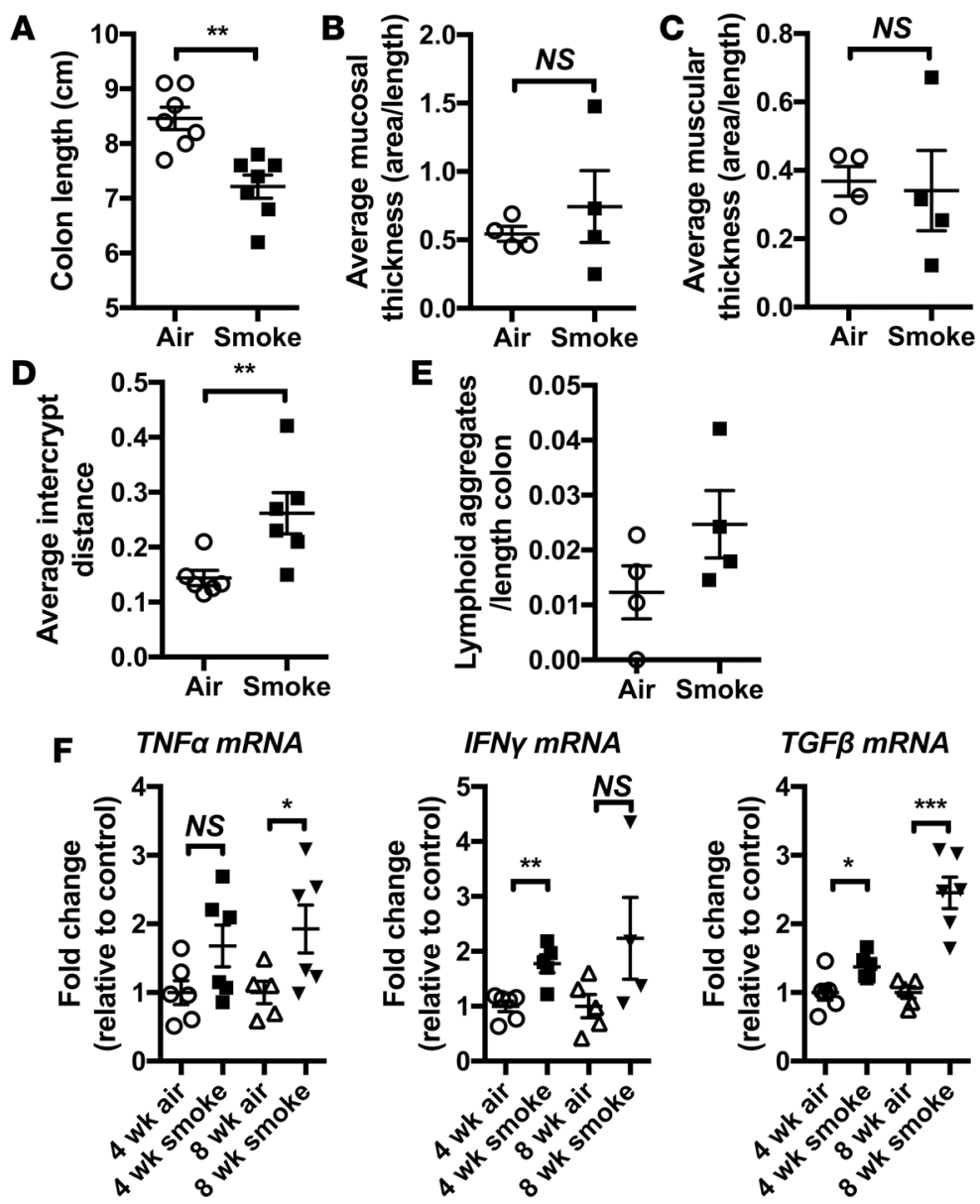

Figure 2. Chronic CS exposure results in mild/subclinical pathology in the colon. Mice were exposed to CS for 4 or 8 weeks. (A) Colons were excised, and length was measured and shown to be significantly shorter, demonstrating that remodeling has occurred in CS-exposed groups compared with normal air-exposed controls $(n=7)$. (B) Thickening of the mucosal layer or (C) the muscular layer underlying the mucosa, commonly observed in experimental colitis models, was not detected in CS-exposed groups $(n=4)$. (D) Gross crypt loss was not evident; however, restructuring of the crypt architecture was evident, with increased intercrypt distance following CS exposure $(n=6)$. (E) Increased numbers of lymphoid aggregates were observed in the mucosal layer of CS-exposed groups $(n=4)$. (F) mRNA levels of TNF- $\alpha$, IFN- $\gamma$, and TGF- $\beta$ were increased in the colons of CS exposed groups ( $n=4-6)$, ${ }^{*} P \leq 0.05$, ${ }^{* *} P$ $\leq 0.01,{ }^{* *} P \leq 0.001$. Student's unpaired 2-tailed $t$ test used for comparisons of 2 groups, 1 -way ANOVA with Tukey's post-hoc was used whenever more than 2 experimental groups were compared.

We also provide evidence that systemic hypoxia may act as a novel driver of intestinal pathologies that underpin the increased incidence and severity of $\mathrm{CD}$ reported in smokers and COPD patients.

\section{Results}

Chronic CS exposure results in lung pathology and impairment of gas exchange. We initially sought to determine whether our mouse model of chronic CS exposure might be useful as a preclinical model for understanding the links between smoking and $\mathrm{CD}$. As expected, and as occurred in our previous studies $(2$, 14-20), mice that were exposed to CS for 8 weeks developed features of experimental COPD, including increased cellular inflammation in the airways (Figure 1A) and lung tissue (Figure 1, B and C); remodeling of small airways, including deposition of collagen and epithelial layer thickening (Figure 1, D and E); emphysema-like alveolar enlargement (Figure 1F); and reduced lung function (Figure 1G) compared with normal air-exposed controls.

For this study we established a potentially novel technique, the diffusing factor for carbon monoxide $\left(\mathrm{DF}_{\mathrm{CO}}\right)$ to assess gas exchange in the lungs (21). Air-exposed control mice had $\mathrm{DF}_{\mathrm{CO}}$ values of $0.750 \pm 0.014$ and $0.758 \pm 0.013$ at 4 and 8 weeks, respectively (Figure 1H). CS-exposed groups had a marked reduction in $\mathrm{DF}_{\mathrm{CO}}(0.631 \pm 0.017$ and $0.479 \pm 0.023$ at 4 and 8 weeks of CS exposure, respectively). Significantly, gas exchange was reduced at 4 weeks, which precedes the onset of significant lung pathology in our model 
A

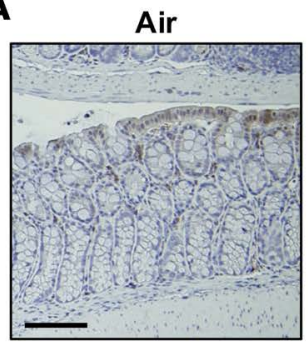

Hypoxyprobe
B
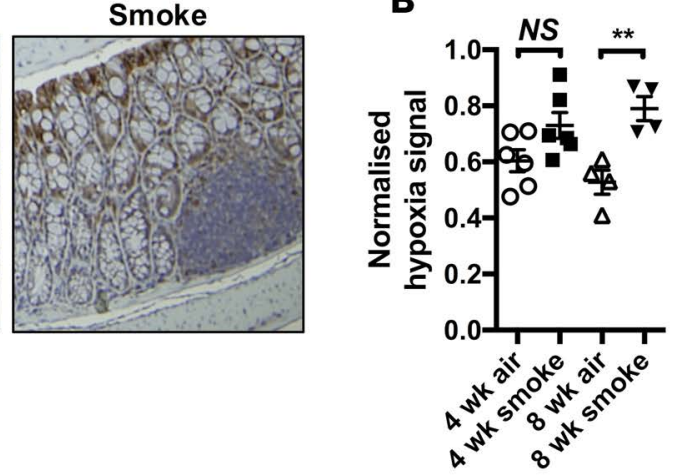

C

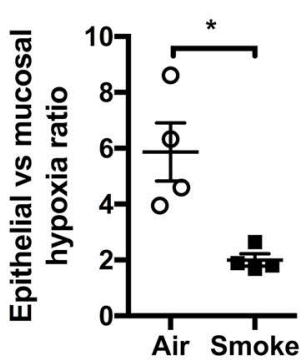

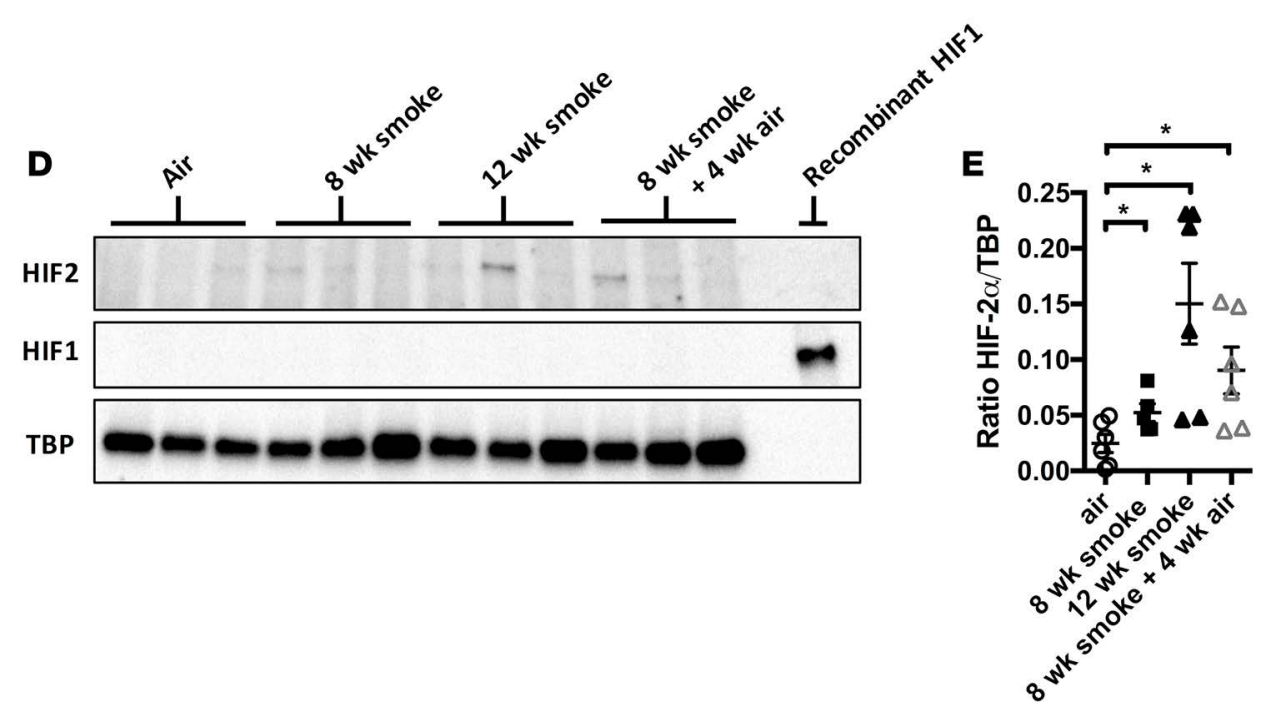

Figure 3. Chronic CS exposure triggers mucosal hypoxia and HIF-2 stabilization. (A) Representative photomicrographs showing colon tissue hypoxia using hypoxyprobe staining in normal air- and CS-exposed colon tissue (scale bar: $100 \mu \mathrm{M}$ ). (B) Increased levels of hypoxia in the colonic mucosal layer normalized to total nuclear material in mucosa was detected in CS-exposed mice $(n=4-6)$. (C) The percentage of epithelial vs. mucosal area that stained positive for hypoxyprobe was determined, and the ratio of these 2 values was calculated, demonstrating a significant shift in hypoxyprobe staining to the mucosal layer of the colon in CS-exposed mice $(n=4)$. (D) HIF-2 $\alpha$ but not HIF-1 $\alpha$ was increased in whole colon tissue lysates from mice exposed to CS for 8 weeks, 12 weeks, or 8 weeks followed by 4 weeks of normal air exposure. (E) Densitometry shows increased HIF-2 $\alpha$ protein relative to TATA-binding protein (TBP) $(n=6) .{ }^{*} P \leq 0.05,{ }^{* *} P \leq 0.01$. Student's unpaired 2-tailed $t$ test used for comparisons of 2 groups, 1-way ANOVA with Tukey's post-hoc was used whenever more than 2 experimental groups were compared.

(14). The greatest suppression of $\mathrm{DF}_{\mathrm{CO}}$ was observed at 8 weeks, which coincides with the onset of the hallmark disease features of experimental COPD. These data suggest that chronic CS exposure progressively decreases the $\mathrm{DF}_{\mathrm{CO}}$ of the lung, including in experimental COPD, and closely resemble the reduced diffusing lung capacity for carbon monoxide $\left(\mathrm{DL}_{\mathrm{CO}}\right)$ observed in healthy smokers and $\mathrm{COPD}$ in humans $(22,23)$.

Chronic CS exposure results in the induction of histological and inflammatory changes in the colon. We then assessed markers of colitis in our model. Colon shortening, a gross indicator of inflammation and tissue remodeling in rodent models of colitis (24), was evident in groups CS-exposed for 8 weeks compared with normal airexposed controls (Figure 2A). Despite this, histological analysis could not detect other typical features of experimental colitis such as mucosal and muscular layer thickening (Figure 2, B and C) (25), although there were significant increases in intercrypt distance (Figure 2D) indicating crypt architecture remodeling. There was also a tendency toward increased numbers of lymphoid aggregates in the colonic tissue (Figure 2E). Concordant with the induction of a mild colitic phenotype similar to that found in humans (26), mRNA expression of CD-associated proinflammatory cytokines TNF- $\alpha$, IFN- $\gamma$, and TGF- $\beta$ was increased in the colon of CS-exposed groups after 4 and 8 weeks (Figure 2F). The TNF- $\alpha$ result did not reach statistical significance at 4 weeks, indicating that these gut changes take time to occur and may not reflect a simple response to CS exposure but may involve CS-induced pathology. Thus, the induction of hallmark features of experimental COPD and impaired gas exchange with CS exposure was accompanied by the induction of a mild colitic phenotype in the colon. 
A

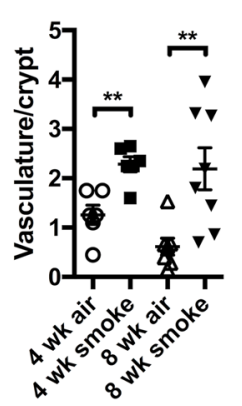

B
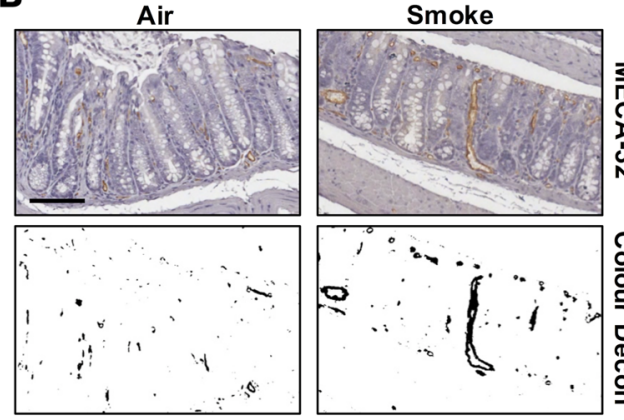

C

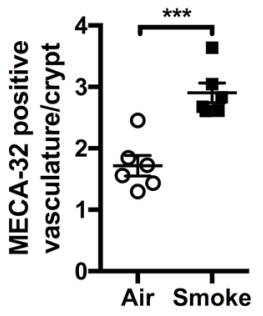

D

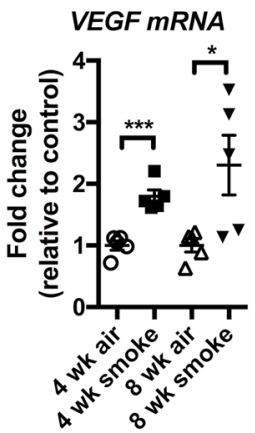

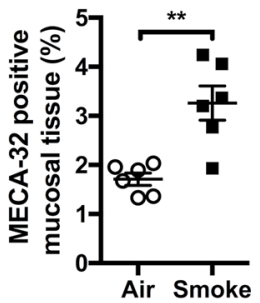

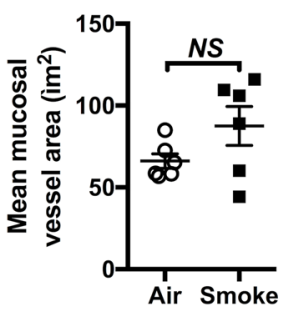

Figure 4. Chronic CS exposure drives vascularization and VEGF expression. Mice were exposed to CS for 4 or 8 weeks. (A) Increased mucosal vasculature was observed in the colons of CS-exposed groups after 4 and 8 weeks of CS exposure $(n=$ 6-8). (B) Vasculature was visualized by IHC against the MECA-32 antigen (upper panels) and color deconvolution performed for quantitative analysis (lower panels) (scale bar: $100 \mu \mathrm{M}$ ). (C) The total number of discrete MECA-32-positive events increased with smoke exposure, as did the total proportion of mucosal tissue that was MECA-32 positive. There was a nonsignificant trend toward increased MECA-32 event area following smoke exposure $(n=6)$. (D) Increased vasculature was accompanied by elevated mRNA expression of proangiogenic factors VEGF and iNOS in whole colon tissue $(n=5-6) .{ }^{*} P \leq 0.05,{ }^{* *} P \leq 0.01,{ }^{* *} P \leq 0.001$. Student's unpaired 2-tailed $t$ test used for comparisons of 2 groups, 1-way ANOVA with Tukey's post-hoc was used whenever more than 2 experimental groups were compared.

Chronic CS exposure is associated with increased colonic tissue hypoxia. Full-thickness mucosal tissue hypoxia and altered mucosal microvasculature are common features of IBD (27-29). To investigate whether increased systemic and colon tissue hypoxia was associated with experimental COPD, we employed the pimanidazole-based dye, hypoxyprobe, which forms immunochemically detectable adducts with thiol-containing amino acids in hypoxic tissues (partial pressure of oxygen $\left[\mathrm{pO}_{2}\right]<10 \mathrm{~mm} \mathrm{Hg}$ ) (30). In colons from normal air-exposed groups, tissue hypoxia was restricted to the luminal epithelial layer (Figure 3, A-C), as previously described $(24,31)$. In contrast, colonic tissue from CS-exposed groups exhibited an increased amount of total hypoxyprobe signal, which was present throughout the mucosal layer, as opposed to being restricted exclusively to the lumenal epithelium.

Tissue hypoxia can drive the stabilization of HIF proteins HIF-1 $\alpha$ and HIF-2 $\alpha$, which subsequently orchestrate tissue-adaptive responses primarily through the regulation of gene networks. We performed immunoblot analysis to test whether HIF-1 $\alpha$ or HIF- $2 \alpha$ were stabilized after 8 weeks of CS exposure. While HIF-1 $\alpha$ was not detectable in any samples tested (Figure 3D), we observed that HIF-2 $\alpha$ protein was stabilized in colon tissue extracts from mice after 8 weeks of CS exposure compared with normal air-exposed controls (Figure 3, D and E).

Chronic CS exposure is associated with increased colonic mucosal vascularization and local expression of angiogenic factors. Angiogenesis can be induced in tissues following hypoxia-mediated stabilization of HIF proteins as part of the adaptive response (32). We undertook a general histological evaluation of colons after 4 and 8 weeks of CS exposure in mice and found that the mucosal vasculature was more extensive compared with those exposed to normal air (Figure 4A). To formally assess this, we performed immunohistochemistry to visualize mucosal vasculature with the panendothelial cell antigen MECA-32 (33) after 8 weeks of CS exposure (Figure 4B). Analysis revealed a higher frequency of MECA-32-positive events per crypt and overall increases in the percentage of mucosal tissue that stained positive (Figure 4C). Mean MECA-32-positive 

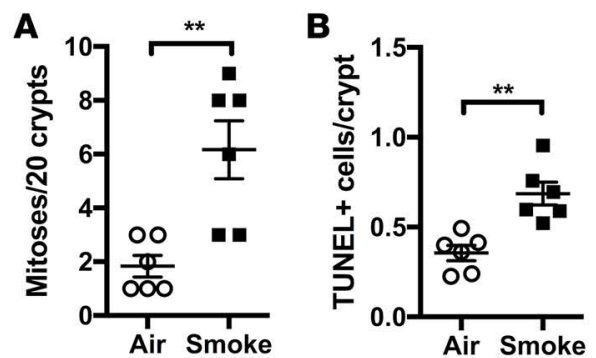

C

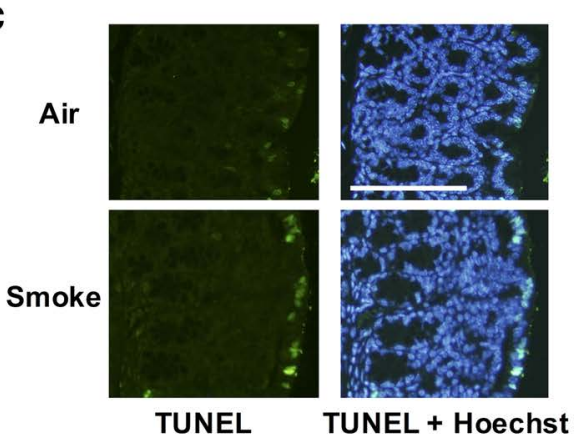

D

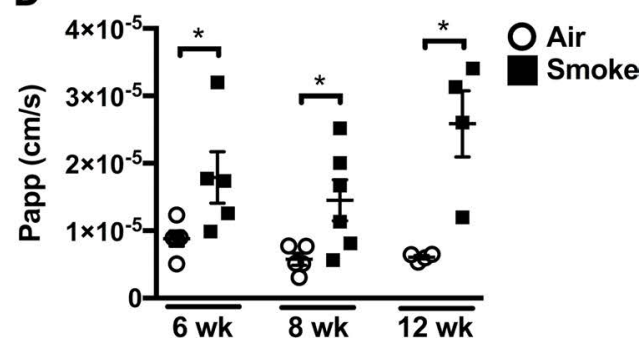

Figure 5. Chronic CS exposure induces altered cellular turnover and increased barrier permeability in the colon. Mice were exposed to CS for 4,8 , or 12 weeks to drive development (4 weeks), establishment ( 8 weeks), and progression (12 weeks) of experimental COPD. (A) After 8 weeks, increased numbers of mitotic nuclei were observed in the colonic crypts of CS-exposed groups $(n=6)$. (B) TUNEL staining was performed to evaluate the extent of cell death, and increased numbers of TUNEL-positive cells were observed in the epithelial and mucosal layers of colons from CS-exposed groups $(n=6)$. (C) Representative fluorescent microscopy images of TUNEL-stained colon tissue. Notably, high numbers of TUNEL-positive cells were observed in the epithelial layer of the colons of CS-exposed groups (scale bar: $100 \mu \mathrm{M}$ ). (D) Increased colon barrier permeability was observed after 8 and 12 weeks of CS exposure $(n=4-6)$. ${ }^{*} P \leq 0.05,{ }^{*} P \leq 0.01$. Student's unpaired 2 -tailed $t$ test used for comparisons of 2 groups, 1-way ANOVA with Tukey's post-hoc was used whenever more than 2 experimental groups were compared.

mucosal vessel area was also increased, although not to a statistically significant extent. Exaggerated vascularization of the CS-exposed colon was accompanied by increases in the expression of mRNA of proangiogenic VEGF and inducible nitric oxide synthase (iNOS), both of which are HIF-inducible target genes (34), after 4 and 8 weeks of CS exposure (Figure 4D).

Chronic CS exposure is associated with altered cellular turnover and decreased barrier function in colons. Tissue hypoxia and cytokines such as TNF- $\alpha$ are known triggers of cell death and turnover in the GIT $(13,35)$. Histological evaluation revealed that chronic CS exposure resulted in increased numbers of mitotic cell profiles in colon crypts compared with normal air-exposed controls (Figure 5A). To quantify cell death in tissues, TUNEL analysis was performed, which revealed an approximately 2-fold increase in the number of dead cells in the colons of CS-exposed groups (Figure 5B). The majority of these dead cells were located within the epithelial layer (Figure 5C). Increased death and damage of the epithelial layer is known to compromise epithelial barrier function (36), and we hypothesized that the altered cellular turnover that was apparent in colons from CS-exposed mice could contribute to decreased barrier function. Importantly, intestinal permeability assays showed that CS exposure increased intestinal permeability in the colon (Figure 5D). Interestingly, increased colon permeability developed in a time-dependent manner and progressively increased after 8 or 12 weeks of CS exposure. Thus, increased colon barrier permeability was preceded by impaired gas exchange in the lungs (Figure $1 \mathrm{H}$ ), mucosal hypoxia (Figure 3, A-C), inflammation (Figure 2, E and F), and angiogenesis (Figure 4, A-D) in the colon after 4 weeks. Furthermore, it developed with prolonged CS exposure (8-12 weeks) in tandem with the induction of COPD-like lung pathology (14-20).

Chronic CS-induced pathological changes in the GIT extend to the ileum. CD affects all areas of the GIT, in particular the ileum and colon, and smoking has been linked with increased incidence of ileal pathology in CD patients $(37,38)$. Thus, we next assessed whether chronic CS exposure of mice for 8 weeks resulted in development of pathological changes in the ileum, like we observed in the colon. Chronic CS exposure was associated with increased levels of tissue hypoxia in ileal tissue (Figure 6A). CS exposure and GIT mucosal tissue hypoxia were again also associated with measurable pathophysiologic changes in the ileum that were 

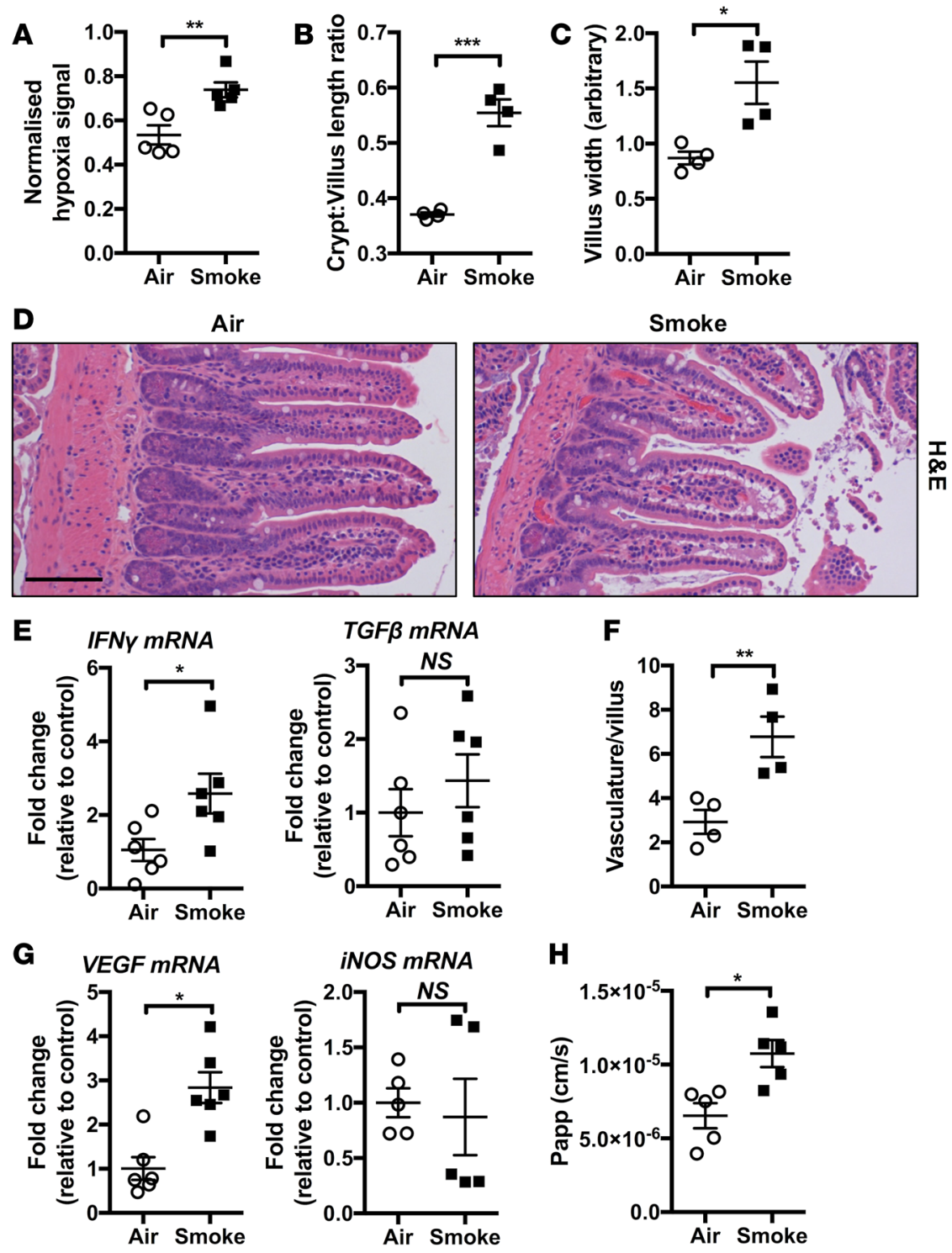

H

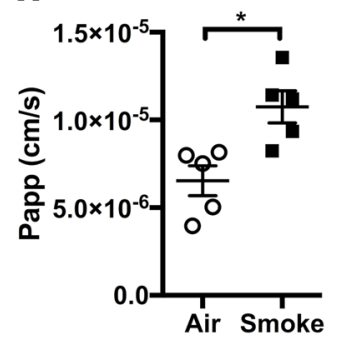

Figure 6. Chronic CS-induced pathology extends to the ileum. Mice were exposed to CS for 8 weeks to induce experimental COPD. (A) Increased total mucosal hypoxyprobe signal was observed in the mucosal layer of ileum of CS-exposed groups $(n=5)$. (B and C) Increased crypt/villus length ratio and villus width in ileum of CS-exposed mice $(n=4)$. (D) Representative photomicrographs of H\&E-stained ileum from normal air- and CS-exposed mice showing altered villus architecture and increased vasculature in CS-exposed groups (scale bar: 100 $\mu M)$. (E) qPCR analysis of inflammatory genes indicated that IFN- $\gamma$ mRNA was increased in the ileum of CS-exposed groups, while TCF- $\beta$ expression was unaltered $(n=6)$. (F) Vasculature and (G) VEGF but not iNOS mRNA were increased in the ileum of CS-exposed groups ( $n$ = 4-6). (H) Reduced epithelial barrier function of the ileum was observed in CS-exposed groups $(n=5) .{ }^{*} P \leq 0.05,{ }^{* *} P \leq 0.01,{ }^{* *} P \leq 0.001$. Student's unpaired 2-tailed $t$ test used for comparisons of 2 groups, 1-way ANOVA with Tukey's post-hoc was used whenever more than 2 experimental groups were compared.

indicative of a mild disease state, including increased ratio of crypt/villus length (Figure 6B) and villus widening (Figure 6, C and D) compared with normal air-exposed controls. mRNA expression of inflammatory cytokines such as IFN- $\gamma$ and TGF- $\beta$ was also increased (Figure $6 \mathrm{E}$ ), while TNF- $\alpha$ expression was unaffected (data not shown). Increased vascularization of villi was also present (Figure 6, D and F). Vascularization was accompanied by increased mRNA expression of the angiogenic factor VEGF, although - in contrast to the colon - the expression of iNOS was unaffected (Figure 6G). Ileum barrier permeability was also increased (Figure 6H). Thus, chronic CS exposure, impaired lung gas exchange, and the induction of a COPD-like phenotype in mice resulted in the induction of a pathological profile in the GIT that extended from the colon into the ileum.

Smoking cessation has differential effects on pathological changes in the colon and ileum. In smokers, the cessation of smoking reduces the incidence and severity of $\mathrm{CD}$ (39). How smoking cessation affects CD incidence and severity in COPD patients is not understood. To explore this, we exposed mice to CS for 12 weeks, at which time COPD-like lung pathology has further developed compared with 8-week exposure (14). Other groups were CS-exposed for 8 weeks, followed by smoking cessation and 4 weeks of normal air exposure. We previously demonstrated that COPD-like pathology such as emphysema persists following this smoking cessation period, as does the increased presence of macrophages in the airways (14). In the colon, the persistence of changes in immune and vascular-related factors varied following smoking cessation. After smoking cessation, TNF- $\alpha$ expression continued to increase, IFN- $\gamma$ expression returned to baseline levels, 
A TNFa mRNA
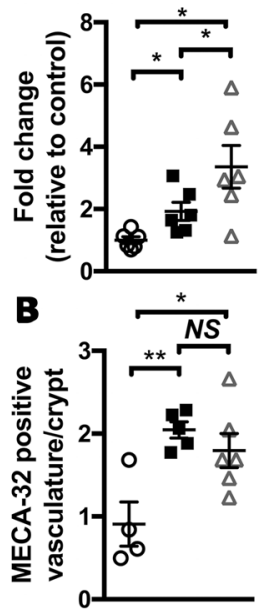

C VEGF IRNA
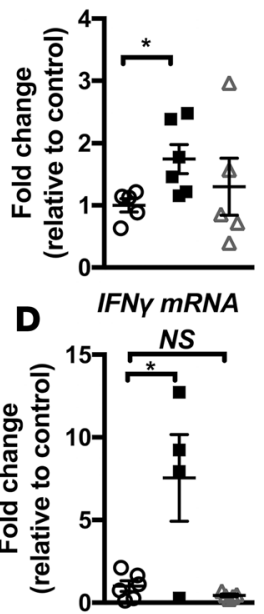

E

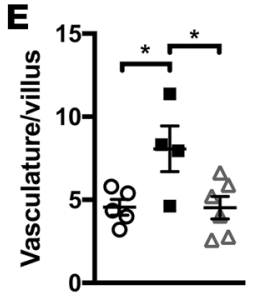

IFNY TRNA
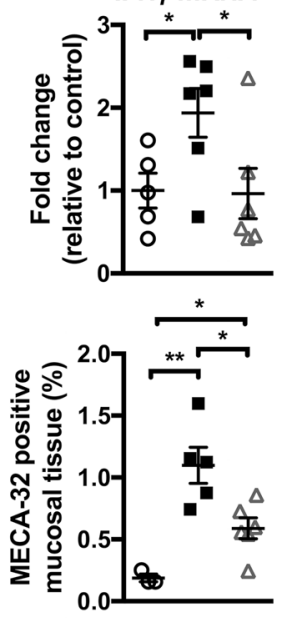

iNOS mRNA

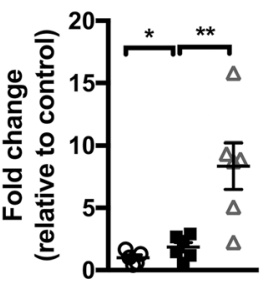

TGF $\beta$ MRNA

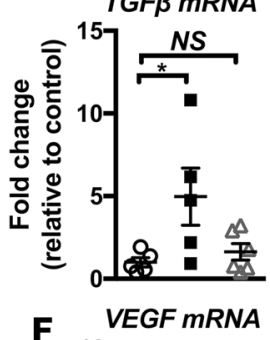

TGF $B$ MRNA
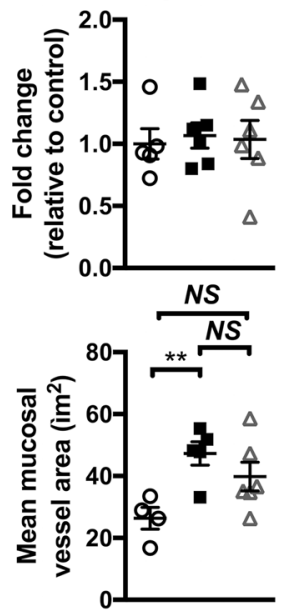

KEY:

O 12 wk Air

12 wk Smoke

$\triangle 8$ wk Smoke

+4 wk Air

Figure 7. Smoking cessation alters pathology in the colon and ileum.

Mice were exposed to CS for 8 weeks to induce experimental COPD and then breathed normal air or continued CS exposure for 4 weeks. (A) qPCR assessment of mRNA showed that TNF- $\alpha$ expression increased, IFN- $\gamma$ expression decreased, and TGF- $\beta$ expression was unaltered in whole colons with smoking cessation $(n=5-6)$. (B) Smoking cessation resulted in nonsignificant decreases in the number of and volume of blood vessels, and a significant decrease in total MECA-32-positive mucosal area in colons compared with groups continually exposed to CS for 12 weeks $(n=$ 4-6). (C) VEGF mRNA was increased in the colon following 12 weeks but not following smoking cessation, while iNOS was significantly increased in the colon following smoking cessation $(n=5-6)$. (D) mRNA levels of IFN- $\gamma$ and TCF- $\beta$ returned to baseline in the ileum following smoking cessation $(n=4-6)$. (E) Smoking cessation reversed the increased vascularization of ileal tissue $(n=4-6)$. (F) VEGF and iNOS expression were increased in the ileum of the 12-week CS-exposed group but not following smoking cessation $(n=4-6)$. ${ }^{*} P \leq 0.05,{ }^{* *} P \leq 0.01$. Student's unpaired 2 -tailed $t$ test used for comparisons of 2 groups, 1-way ANOVA with Tukey's post-hoc was used whenever more than 2 experimental groups were compared.

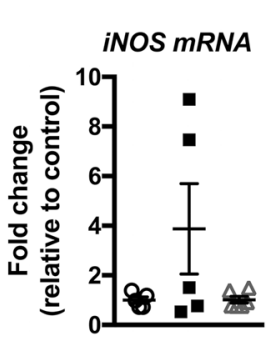

and TGF- $\beta$ was not upregulated after 12 weeks of smoking or following smoking cessation (Figure 7A). HIF- $2 \alpha$ stabilization remained evident after 12 weeks of CS exposure and was still present after smoking cessation (Figure 3D). Consistent with this, smoking cessation reduced the total amount of mucosal vascular tissue compared with continued CS exposure, although vascular area and density remained elevated compared with normal air-exposed controls (Figure 7B). VEGF expression decreased following smoke cessation, although not consistently; however, iNOS expression significantly increased (Figure 7C), confirming an altered inflammatory environment in the colon following smoking cessation. In contrast, CS-induced pathology of the ileum resolved following smoking cessation, with the levels of expression of IFN- $\gamma$ and TGF- $\beta$ (Figure 7D), vascularisation of villi (Figure 7E), and VEGF and iNOS expression (Figure 7F) all returning to air-exposed control levels. Thus, with smoking cessation, some inflammatory and pathological features remained or progressed in the colon, while most CS-associated changes resolved in the ileum.

The pan-PHDi DMOG rescues chronic CS-induced GIT pathology independently of lung pathology. Tissue hypoxia is an important feature of colitis and has been shown to trigger epithelial layer damage and increased permeability in the GIT (29). Our data suggest a scenario whereby the combination of CS exposure and reduced 
A

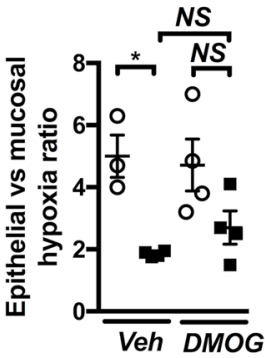

C

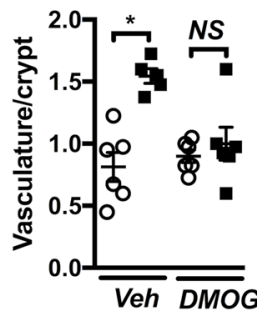

E

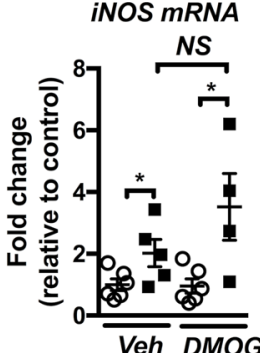

$\mathbf{F}$

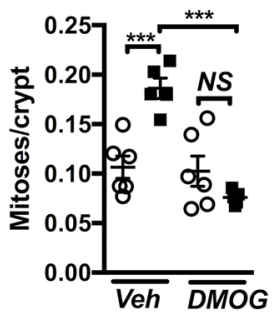

TNFa mRNA
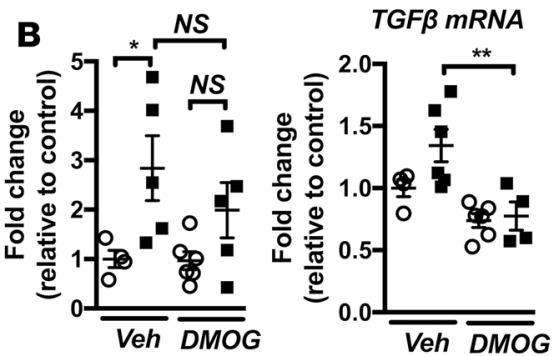

VEGF TRNA
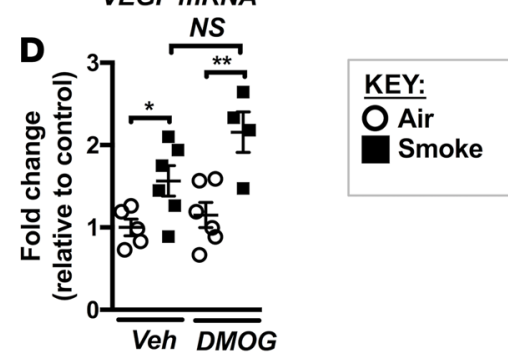

Figure 8. DMOG treatment during chronic CS exposure prevents pathology in the colon. Mice were exposed to CS for 8 weeks to induce experimental COPD and were treated with DMOC throughout. (A) Tissue hypoxia was measured using hypoxyprobe, and DMOG treatment partially prevented the mucosal hypoxic phenotype in colons of CS-exposed mice $(n=3-4)$. (B) DMOC treatment partly prevented increases in TNF- $\alpha$ and TGF- $\beta$ mRNA expression in the colon $(n=4-6)$. (C) DMOG treatment inhibited increases in vasculature $(n=5-6)$. ( $D$ and $\mathbf{E})$ DMOG treatment increased the mRNA expression of HIF target genes VEGF, iNOS, CD73, and gravin in the colons of CS-exposed groups $(n=4-6)$. (F) DMOC treatment completely inhibited increases in crypt mitoses $(n=$ 5-6). (G) DMOG treatment completely inhibited the CS-induced increases in colon barrier permeability in CS-exposed mice ( $n$ $=4-6) .{ }^{*} P \leq 0.05,{ }^{* *} P \leq 0.01,{ }^{* *} P \leq 0.001$. Student's unpaired 2-tailed $t$ test used for comparisons of 2 groups, 1-way ANOVA with Tukey's post-hoc was used whenever more than 2 experimental groups were compared.
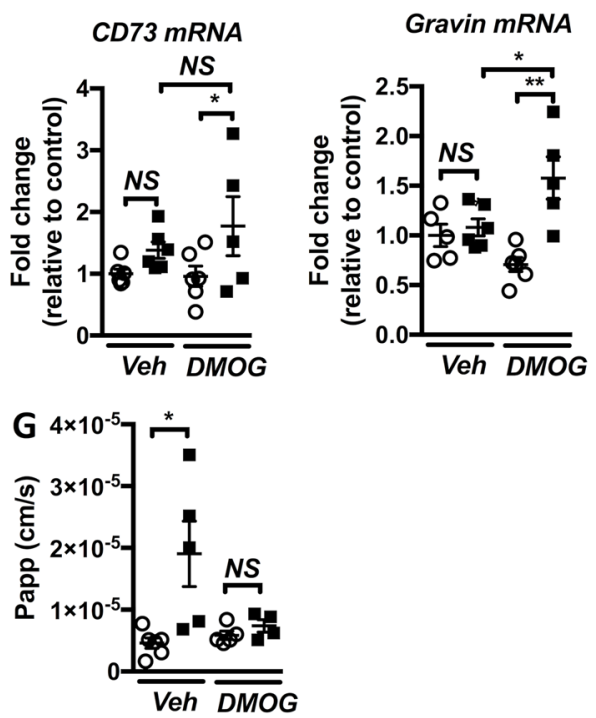

gas exchange and lung function in experimental COPD may result in the development of a chronic hypoxic state in the GIT. This may lead to the activation of HIF-2-associated signaling and attempted compensatory vascularization of the affected mucosal tissue. The data presented above show that, despite increased vascularization, elevated tissue hypoxia persisted in the mucosal layer, which could be an important driver of cell death, inflammation, and increased intestinal permeability in the GIT following CS exposure (Figure 2-7). Thus, we examined the effects of suppressing tissue hypoxia and increased barrier permeability, and therefore CD-like pathology, with a targeted pharmacological intervention. Prolyl hydroxylase domain inhibitors (PHDis) have been investigated as potential therapeutic agents in IBD and have protective effects in experimental models of colitis, including the prevention of colitis-associated increased gut permeability $(24,25)$. To assess the therapeutic potential of PHDis in CS-induced CD-like features, we systemically (i.p.) treated mice with the pan-PHDi dimethyloxaloylglycine (DMOG) during the course of 8 weeks of CS exposure and examined the effects on lung and GIT pathology. DMOG treatment had no significant effect on cellular and molecular inflammation or pathology in the lung induced by chronic CS exposure. There were no effects on CS-mediated increases in leukocyte, macrophage, neutrophil, and lymphocyte influx into the airways (Supplemental Figure 1, A-D; supplemental material available online with this article; https://doi.org/10.1172/ jci.insight.94040DS1); upregulation of key macrophage and neutrophil chemokines MCP-1 and CXCL1; 

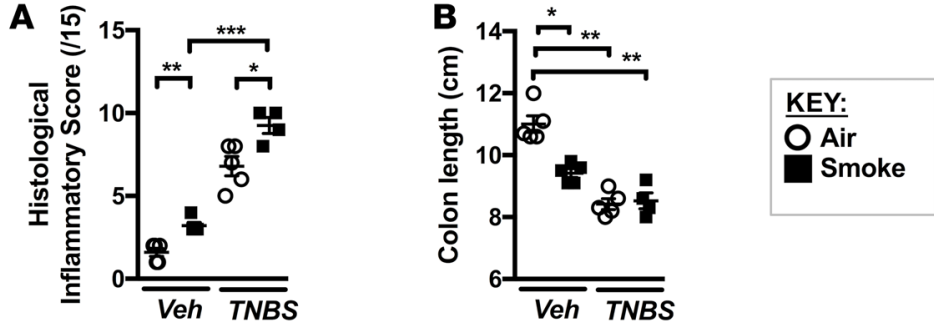

\section{Air TNBS}

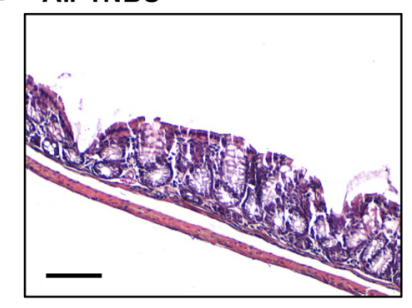

\section{Smoke TNBS}

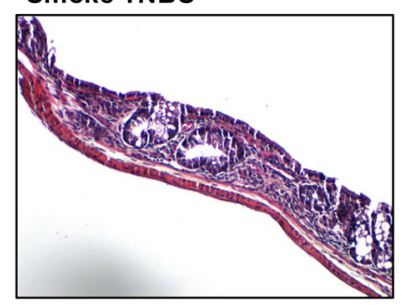

Figure 9. Chronic CS exposure predisposes to TNBS-induced experimental colitis. Mice were exposed to CS for 4 weeks, followed by the induction of colitis with TNBS. (A) Histological inflammatory scoring of $\mathrm{H} \& \mathrm{E}$ sections revealed that CS exposure resulted in a more severe colitic phenotype in response to TNBS, although (B) a further shortening of the colon did not occur $(n=4-5)$. (C) Representative micrographs of $\mathrm{H} \& \mathrm{E}$-stained colon sections from normal air-exposed TNBS-treated and CS-exposed TNBS-treated mice. Colons from CS-exposed TNBS-treated mice displayed greater mucosal inflammatory infiltration and crypt dysplasia (scale bar: $100 \mu \mathrm{M})$. (D) Expression of iNOS, VEGF, TGF- $\beta$, and TNF- $\alpha$ in mice exposed to normal air or CS \pm vehicle or TNBS $(n=3-6)$. ${ }^{*} P \leq 0.05,{ }^{*} P$ $\leq 0.01,{ }^{* *} P \leq 0.001$. Student's unpaired 2-tailed $t$ test used for comparisons of 2 groups, 1-way ANOVA with Tukey's post-hoc was used whenever more than 2 experimental groups were compared.
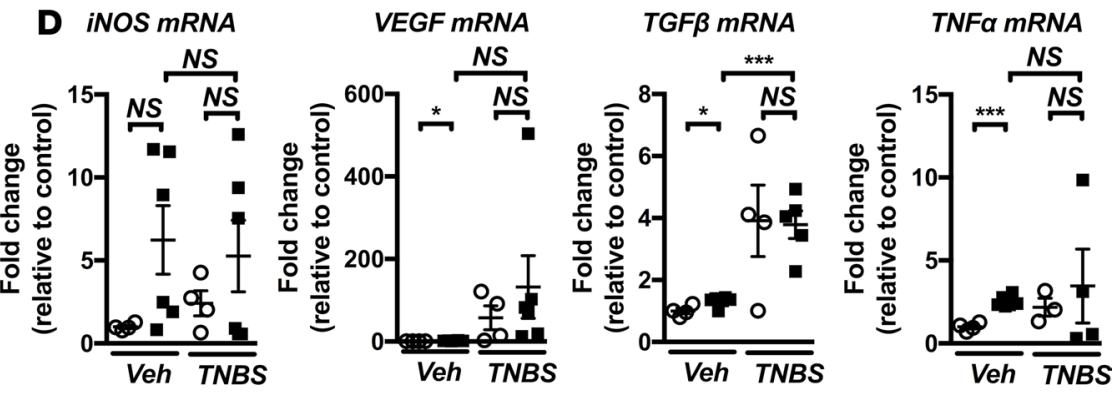

or matrix metalloprotease (MMP)12 levels (Supplemental Figure 1E). CS-induced emphysema-like alveolar enlargement was also unaffected by DMOG treatment (Supplemental Figure 1F). In contrast, examination of the altered hypoxyprobe staining in the colon revealed that, in most mice, DMOG prevented the significant reduction in CS-related hypoxia in the mucosal layer relative to the epithelial layer, compared with the vehicle-treated group, and there was a nonsignificant trend toward protection compared with the vehicle-treated CS-exposed group (Figure 8A). DMOG treatment prevented the increases in expression of CD-associated inflammatory genes TNF- $\alpha$ and TGF- $\beta$ in CS-exposed mice (Figure 8B). In addition, the increased vasculature that characterized CS-exposed colonic mucosal tissue was also not present in DMOG-treated mice (Figure 8C). Despite this, DMOG treatment further upregulated colonic VEGF expression in CS-exposed groups (Figure 8D). As VEGF is an HIF-1 and HIF-2 target gene, it was not unexpected that DMOG treatment would further increase its expression; indeed, the expression of other known HIF-1 target genes such as iNOS, CD73, and gravin were also increased (Figure 8E). DMOG treatment was associated with complete protection against altered crypt cell turnover (Figure 8F). Most importantly, DMOG treatment completely prevented CS-induced increases in colon permeability (Figure 8G). In summary, systemic treatment with the PHDi DMOG suppressed colon hypoxia and associated pathology in CS-induced experimental COPD.

Smoke exposure predisposes to more severe TNBS-induced colitis. Tobacco smokers have a higher incidence of $\mathrm{CD}$, as well as worse disease progression, compared with nonsmokers (6). The heightened risk of CD observed in smokers is further elevated in COPD patients. Our data indicate that chronic smoke exposure resulted in a range of pathological changes to the GIT, and we hypothesized these changes would sensitize to a subsequent colitis-inducing challenge. To test this, we assessed the response of CS-exposed mice to a well-characterized model of 2,4,6-trinitrobenzenesulfonic acid-induced (TNBS-induced) experimental colitis (40). It is likely that mice with experimental COPD would not survive a TNBS challenge. Thus, we exposed mice to CS for 4 weeks, at which time gas exchange in the lung was impaired and many pathological GIT changes were observed (Figures 1-4), and then challenged them with TNBS while ceasing smoke exposure. As expected, TNBS caused a marked induction of CD-like pathology, indicated by increases in histological 
A

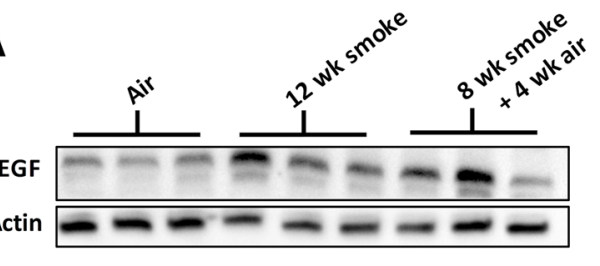

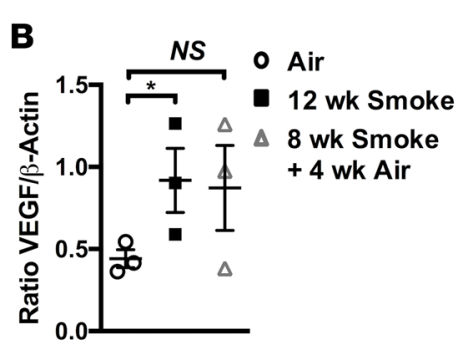

B

Figure 10. Serum VEGF is elevated in mice chronically exposed to CS and in current and former smokers with CD. (A) Mice were exposed to normal air or CS for 12 weeks or CS for 8 weeks followed by 4 weeks normal air. Immunoblotting of serum for VEGF. (B) Densitometric analysis of immunoblotting revealed elevated levels of serum VEGF in CS-exposed groups compared with normal air-exposed controls $(n=3)$. (C) Serum VEGF was elevated in the serum of human CD patients compared with healthy controls $(n=12)$. (D) Serum VEGF is elevated in smokers and ex-smokers compared with never-smokers (both healthy and CD) $(n=8)$. (E) Current or former smokers with CD show significantly elevated serum VEGF $(n=4)$. (F) Total CD34 signal as a percentage of tissue area in surface mucosal biopsies from the terminal ileum of healthy nonsmoker, healthy smoker, CD nonsmoker, and CD smoker patients was quantified $(n=2-3)$. (C) Representative photomicrographs of terminal ileum mucosal biopsies stained for CD34 (scale bar: $50 \mu \mathrm{M}$ ). ${ }^{*} P \leq 0.05,{ }^{*} P \leq 0.01$. Student's unpaired 2 -tailed $t$ test used for comparisons of 2 groups, 1-way ANOVA with Tukey's post-hoc was used whenever more than 2 experimental groups were compared.
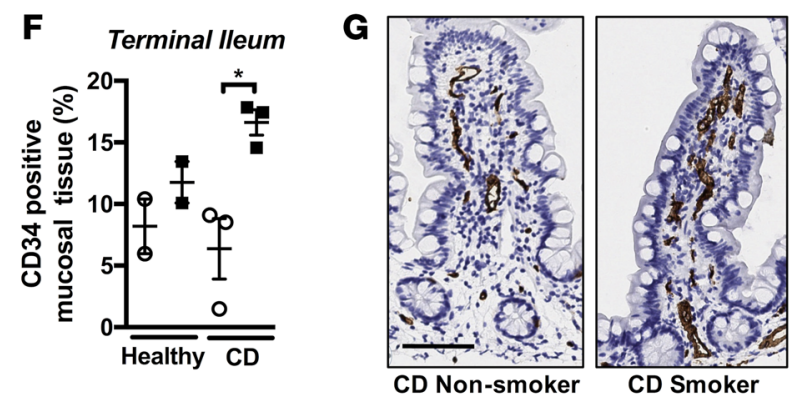

inflammatory score (Figure 9A) and colon shortening (Figure 9B). Prior CS exposure exaggerated the severity of pathology induced by TNBS with further increases in histopathological score (Figure 9, A and C). We also examined the expression of TNF- $\alpha$, TGF- $\beta$, VEGF, and iNOS (Figure 9D), which showed trends toward further increased expression in CS-exposed TNBS-treated mice compared with normal air-exposed controls. Expression of other colitis-associated cytokines IL-6 and IL-12p40 showed a similar trend toward highest expression in CS-exposed TNBS-treated mice compared with other groups (data not shown). Thus, CS exposure predisposed to the development of a more severe phenotype of colitis in our TNBS model, which supports the epidemiological data linking smoking, COPD, and increased CD severity.

Smoking and CD are associated with elevated serum VEGF and GIT mucosal vasculature in humans. Cigarette smoking and associated systemic hypoxia have been linked to elevated peripheral levels of VEGF in the context of lung and liver disease (41-43). We found that serum VEGF levels were increased in mice following chronic smoke exposure and smoking cessation and in association with the development of CD-like features (Figure 10, A and B). We then sought to validate our findings in preclinical mouse models in clinical samples from current smokers, former smokers, and never-smokers with CD compared with subjects who were considered healthy. CD patients had elevated serum VEGF levels compared with healthy participants, as previously reported (Figure 10C) (44). Importantly, both current and former smokers had significantly elevated VEGF levels compared with never-smokers (Figure 10D). When stratified by disease and smoking status, current and former smokers with CD had significantly increased VEGF, with trending increases in 
current and ex-smokers of healthy status (Figure 10E). We also performed histological analysis of mucosal biopsies of ileum taken from healthy or CD patients, stratifying on the basis of smoking status. We observed increased mucosal vascularity in the ileum of smokers with CD compared with nonsmokers, supporting our preclinical model findings (Figure 10, F and G). These data suggest that smoking induces lasting effects on systemic VEGF, a marker of systemic hypoxia, that is highest in current and former smokers suffering from $\mathrm{CD}$ and could contribute to colonic remodeling, as evidenced by increased mucosal vasculature.

\section{Discussion}

Epidemiological evidence suggests that GIT symptoms are highly prevalent among smokers and COPD patients, and that smoking and COPD are associated with higher risks of CD compared with nonsmokers (6). The mechanistic links between smoking and CD are poorly understood, and those linking COPD to IBD have barely been explored. Here, we identify mechanisms by which chronic smoke exposure and smoke-induced lung diseases, such as COPD, influence intestinal integrity and may predispose to chronic intestinal conditions such as CD. Using a preclinical mouse model, we show for the first time to our knowledge that chronic inhalational smoke exposure induces impaired gas exchange and pathology in the lung. This leads to hypoxia in the GIT; stabilization of HIF- $2 \alpha$; ischemic damage in the intestine with colon remodeling, inflammatory responses, and increased microvascular density; and exaggerated barrier permeability. This pathology extends to the ileum, a critical site of CD lesions associated with smoking. Smoking cessation reverses pathology in the ileum and, to a lesser extent, the colon. Importantly, CS-induced GIT pathology could be suppressed by systemic PHDi treatment independently of lung pathology. CS-induced gut pathology primed for and increased the severity of experimental colitis. Our preclinical findings are validated by data from clinical samples that establish a correlation between smoking status, systemic hypoxia, and $\mathrm{CD}$, as well as increased mucosal vasculature in the terminal ileum of $\mathrm{CD}$ patients that smoke. Based on these findings, we propose a model whereby chronic CS exposure, and resulting COPD, impair pulmonary gas exchange resulting in systemic hypoxia. GIT hypoxia and HIF-2 $\alpha$ stablilization drive angiogenesis and epithelial cell damage, impairing epithelial barrier function and setting up a cycle of increasing exposure and inflammatory reaction to lumenal antigens, which ultimately predisposes to and increases the severity of $\mathrm{CD}$. Indeed, there is likely to be a strong influence of gut microbiota in $\mathrm{CD}$ and potentially COPD pathology (11). Gut-lung crosstalk, where changes in the microbiota or inflammatory responses in one organ can affect the other promoting distal inflammation, is now a well-established concept (11). We have observed substantial changes in microbial populations in the gut with CS exposure and the development of experimental COPD (unpublished data). Others have shown that chronic CS exposure of mice altered microbial gut populations and the expression of mucus genes in the colon (45).

We show here, for the first time to our knowledge in a preclinical model of experimental COPD, that impaired pulmonary gas exchange is an early pathological change induced by chronic CS exposure. Impaired gas exchange was evident after 4 weeks of CS exposure, prior to the development of hallmark COPD pathologies such as airway remodeling, emphysema, and impaired lung function that appear after 8 weeks (14). This observation is supported by a recent clinical study showing that smokers with impaired gas exchange but normal spirometry are more likely to develop COPD than smokers with normal gas exchange and spirometry (22). Collectively, these studies suggest that impaired gas exchange may precede the induction of COPD pathology.

Impaired gas exchange induced by smoking has long been associated with systemic hypoxia. Indeed, polycythemia is a well-established consequence of heavy smoking, as the body attempts to compensate for reduced oxygen availability by increasing production of oxygen-carrying RBCs (46-48). Our finding that serum VEGF, a cytokine and marker of systemic hypoxia (41-43), is increased in both preclinical and clinical samples in current and former smokers is suggestive that chronic CS exposure can induce long-lasting systemic effects. Increased serum VEGF in CD has been reported previously (44). Our observations of the effects of current or former smoking in increasing serum VEGF in CD patients (and to a lesser extent healthy patients) supports our hypothesis that smokers that develop impaired gas exchange and systemic hypoxia are at higher risk of developing $C D$. Further studies are required to examine serum in COPD patients with or without $\mathrm{CD}$, where we hypothesize that further impairment of lung function would lead to greater increases in systemic hypoxia.

Our observations of impaired gas exchange in the lung and increased circulating VEGF prompted us to investigate whether intestinal oxygen levels may be perturbed after chronic CS exposure. Histological 
analysis of hypoxia-dependent pimonidazole retention revealed the development of transmural hypoxia in CS-exposed mice, as opposed to the epithelial localization of hypoxia in the normal gut $(24,31)$. In support of this role for smoking-associated GIT hypoxia, laser doppler analysis of smokers demonstrated a decrease in rectal blood flow within 30 minutes of smoking (49). CD is a transmural disease associated with tissue ischemia (28) and maladaptive angiogenesis $(27,50)$, and it has been proposed that the cyclic nature of GIT inflammation in CD can lead to incomplete neovascularization, hindering recruitment of inflammatory cells and production of inflammatory mediators $(51,52)$. Thus, chronic smoke exposure may accelerate these processes, and indeed, in our animal model, smoking led to a more severe pathology in a subsequent TNBS model of colitis.

Tissue hypoxia can result in stabilization of HIF proteins HIF-1 $\alpha$ and HIF-2 $\alpha$, which orchestrate adaptive responses to hypoxia through transcriptional activation of target genes. It is increasingly recognized that HIF- $1 \alpha$ and HIF-2 $\alpha$, although sharing redundant functions, are distinct in their modes of activation, the downstream signaling they engage, and the biological outcomes of the responses they mediate (53). HIF-1 $\alpha$ becomes acutely activated during episodes of severe hypoxia, and its activity does not persist beyond the initial hypoxic insult, while HIF-2 $\alpha$ appears to be activated under conditions of mild or physiological hypoxia in a prolonged manner. This distinct activation profile fits with a model based on our findings whereby gradual deterioration of lung function over a long period of CS exposure results in chronic and mild systemic and tissue hypoxia, resulting in selective activation of HIF-2 $\alpha$. HIF-1 $\alpha$ and HIF-2 $\alpha$ also appear to regulate overlapping and distinct gene networks, and our observation that VEGF (but not other known HIF-1 $\alpha$ targets such as CD73 and gravin) is induced in the colons of CS-exposed mice fits with prior observations that HIF-2 $\alpha$ is the dominant isoform in the regulation of VEGF (54). Selective activation of HIF-2 $\alpha$ observed in the colons of CS-exposed mice also provides a potential mechanistic explanation for the link between smoking and colorectal cancer (55), as HIF-2 $\alpha$ may have roles in promoting tumorigenesis (54).

The GIT is highly susceptible to ischemia, largely due to the nature of oxygen exchange in the villous microvascular system, which results in a steep descending oxygen gradient from the villus base to the tip (56). Thus, intestinal ischemic disease is commonly associated with diseases that limit blood flow, such as chronic renal failure and atherosclerosis (57). Angiogenesis in colitis is linked to increased expression of VEGF (58). VEGF-targeting therapies suppress angiogenesis and inflammation in animal models of colitis (59). While we found that VEGF was upregulated in the serum and GIT following chronic CS exposure, pharmacological intervention with DMOG prevented angiogenesis while further increasing the expression of HIF target VEGF. Thus, experimentally, the upregulation of VEGF signaling does not appear to be sufficient to drive angiogenesis, and other as yet unidentified factors may play a role, possibly in combination with VEGF. Regardless of the role of VEGF, our observations demonstrating increased vascularization associated with smoking status in human CD ileal biopsy samples validate our findings in our preclinical model and suggest that aberrant angiogenesis is a key feature of smoking-associated GIT disease.

Epithelial apoptosis and increased permeability are pathologies commonly associated with intestinal ischemia (60). Our data indicate that colonic changes such as hypoxia and angiogenesis occurred early, along with the impairment of gas exchange in the lung, but that intestinal barrier dysfunction developed concurrently with the establishment of pulmonary pathology induced by chronic CS exposure and was not present after 4 weeks of CS exposure. After 4 weeks of CS exposure, inflammation is firmly established in the lungs; however, hallmark pathologies such as airway remodeling and emphysema have not yet developed (14). We propose 2 possible explanations for this apparent delay in onset of GIT barrier dysfunction relative to other pathologies: that chronic impairment of gas exchange resulting in GIT hypoxia and pathology may result in accumulating epithelial damage and delayed intestinal barrier dysfunction, or alternatively, that more severe lung pathology is needed to induce it, rather than inflammation or CS alone. In support of the latter hypothesis, Rutten and colleagues recently described a link between COPD and IBD demonstrating in a small controlled patient group that COPD patients displayed increased gut permeability at rest, which was further exacerbated after mild exertion along with markers of systemic anaerobic metabolism (13). Collectively, these studies suggest that impaired gas exchange associated with smoking and COPD result in exaggerated systemic hypoxia, which can drive or further exacerbate intestinal barrier dysfunction.

Epithelial cell properties and fate are important determinants of intestinal barrier function, and exaggerated epithelial cell death appears sufficient to trigger severe intestinal inflammation (61). Important in the context of $\mathrm{CD}$, a previous history of smoking is a major risk factor for disease in late-onset $\mathrm{CD}$. While CD generally presents in young adults, emphysema and COPD are diagnosed much later; however, 
impaired gas exchange and subclinical pulmonary damage induced by smoking may manifest much earlier. The threshold of lung damage required to induce GI pathology has not been defined. However, population analysis has identified an increased risk for IBD in COPD patients (8). Smoking-induced barrier dysfunction provides a potential mechanism for other extrapulmonary pathologies associated with smoking. Smokers are at increased risk of nonalcoholic fatty liver disease (NAFLD), and CS exposure also increases the severity of established disease $(62,63)$. Importantly, increased mucosal permeability is thought to be an initiating factor in NAFLD, and patients have demonstrated both reduced epithelial barrier function and endotoxemia (64). Similarly, endotoxin has been shown to induce an initiating pancreatic injury in murine models of alcohol-induced chronic pancreatitis (65), and current smokers are at an increased risk of developing this disease $(66,67)$. Thus, smoking-associated increases in GIT mucosal permeability may contribute to the initiation or exacerbation of these chronic diseases.

The decline in gas exchange capability and pulmonary function due to smoke exposure is currently irreversible, and smoking cessation merely delays or halts the progression of COPD. While smoking cessation reverts the risk of developing $\mathrm{CD}$ to baseline levels in general, the effects of smoking cessation on $\mathrm{CD}$ risk in COPD patients has not been reported (8). In our model, smoking cessation following the establishment of experimental COPD ameliorated GI pathology in the colon and ileum to different extents. In the colon, cessation led to reduction in IFN- $\gamma$ mRNA and partially reduced vasculature, but it was associated with sustained HIF- $2 \alpha$ stabilization and increased TNF- $\alpha$ and iNOS expression. In the ileum, cessation led to the resolution of inflammation and microvascular density. Incomplete inflammatory resolution in the colon may be due to the difference in basal oxygen tensions between the colon and ileum (68), as well as the added metabolic stress of the colonic microbiota (69). Moreover, the tendency toward a greater resolution of ileal pathology following smoking cessation may be clinically reflective of the ileal improvement in the majority of CD patients that benefit from smoking cessation (39).

Pharmacological activation of HIF-associated adaptive hypoxia responses is a promising experimental strategy for the treatment of IBD $(25,40)$. While many studies of these PHDi compounds employ targeted delivery and selective activation pathways, we have previously shown that the panPHDi DMOG acts systemically, increasing erythropoietin (EPO) and hematocrit, while also offering localized protection against inflammation in the GIT (25). Administration of DMOG during CS exposure partially prevented the COPD-associated mucosal hypoxic phenotype and reduced increases in microvascular density, epithelial cell turnover, and impairment of barrier function. While colon mucosal layer hypoxia was reduced, systemic treatment did not affect pulmonary features in experimental COPD. Thus, the prevention of colon mucosal hypoxia was unlikely to be due to a protective effect of DMOG in the lung, but it may reflect an increased oxygen carrying capacity of the blood of treated mice, resulting in more effective perfusion of the colonic tissue. Increased expression of hypoxia-adaptive responses may also account for the GIT-protective effects of DMOG in experimental COPD, and further studies will be required to test this. A prior study demonstrated selectivity of DMOG toward HIF-1 $\alpha$ stabilization over HIF-2 $\alpha$, which may provide an additional mechanism whereby DMOG exerts barrier-protective effects in our model (70). Additional studies will also be needed to assess the local benefits of suppressing hypoxia in the lung on COPD pathogenesis.

Many other mechanisms could be involved in the links between pulmonary pathology and the GIT. These could involve other systemic responses such as conditioning of the BM, widespread and transportable inflammatory factors such as microRNAs and inflammasomes/ASC specks could be involved, and events may be exaggerated by infectious exacerbations of pulmonary disease (16, 71-73). These potential pathogenic processes could be examined using combinations of animal models and clinical studies.

In summary, we have demonstrated a potentially novel mechanism for the development of pathophysiologically relevant alterations in the colon and ileum during chronic inhalational CS exposure and development of COPD, reflective of clinical observations. The CS-induced impaired gas exchange and pulmonary pathology may promote systemic hypoxia, along with the induction of intestinal hypoxia, inflammation, mucosal angiogenesis, and epithelial cell death and reduced intestinal barrier function, which are all likely to contribute to increased susceptibility to CD. Our results support and expand on the limited existing mechanistic data regarding the link between smoking, COPD, and CD and highlight the potential of targeted therapeutic interventions such as hydroxylase inhibitors for the treatment of smoking-associated GIT dysfunction. 


\section{Methods}

Experimental COPD. WT female C57BL/6 mice (Australian BioResources) were exposed to the smoke of 12 cigarettes (3R4F reference cigarettes; University of Kentucky, Lexington, Kentucky, USA) twice/day, 5 times/week for up to 12 weeks as previously described (14-18). An in-house custom-designed and purpose-built nose-only, directed flow inhalation and smoke-exposure system ( $\mathrm{CH}$ Technologies) was used to expose the mice to mainstream CS. Controls were exposed to normal air. For smoking cessation experiments, mice were exposed to CS for 8 weeks and then normal air for a further 4 weeks.

DMOG treatment and TNBS model. The pan-PHDi DMOG (Cayman Chemicals) was dissolved in sterile PBS and administered i.p. ( $8 \mathrm{mg}$ ) every other day prior to CS exposure ( $3 \times$ weekly) as previously described (74). Control animals were administered injections of sterile PBS alone. CS-exposed and control mice were exposed to TNBS as previously described (40).

Airway inflammation, remodeling, and emphysema. Airway inflammation was quantified by enumeration of macrophages, neutrophils, and lymphocytes in bronchoalveolar lavage fluid (BALF) as previously described $(3,14,16-18)$. For histological analysis, lung tissue was fixed in formalin prior to mounting, sectioning, and staining with H\&E for assessment of lung histopathology and emphysema. For analysis of airway remodeling, the thickness of the epithelial layer was measured in airways with a basement membrane perimeter $<1,000 \mu \mathrm{m}(14,19)$. Area of collagen deposition around these airways was quantified using Masson's trichrome-stained lung sections with ImageJ software (NIH) and color deconvolution processing $(15,19,75)$. Emphysema was evaluated using the mean linear intercept technique, a standard method for examining alveolar diameter and damage in rodent models of lung damage $(14-18,76)$.

$D F_{C O}$. A single-breath maneuver to assess $\mathrm{DF}_{\mathrm{CO}}$ was performed as described previously (21). Briefly, mice were anesthetized with ketamine $(117 \mathrm{mg} / \mathrm{kg})$ and xylazine $(14.4 \mathrm{mg} / \mathrm{kg}$, both from Troy Laboratories), a tracheostomy with ligation was performed $(14,19,76)$, and a 19-gauge cannula was inserted into the trachea. Lungs were inflated with $1 \mathrm{ml}$ of tracer gas mixture $(0.318 \% \mathrm{Ne}, 0.302 \% \mathrm{CO}$ and balance air). Following a 9-second breath hold, $1 \mathrm{ml}$ of gas was withdrawn from the lung, diluted to $2 \mathrm{ml}$ (total volume) with room air, and the concentrations of $\mathrm{Ne}$ and $\mathrm{CO}$ were measured by gas chromatography (60-second gas analysis time; INFICON). The $\mathrm{DF}_{\mathrm{CO}}$ is defined as $\left.1-\left(\mathrm{CO}_{9} / \mathrm{CO}_{c}\right) / \mathrm{Ne}_{9} / \mathrm{Ne}_{c}\right)$, where the subscripts 9 and $c$ refer to the concentrations of gas after a 9-second breath-hold time and calibration gas injected, respectively. The $\mathrm{DF}_{\mathrm{CO}}$ is a dimensionless variable expressed as a value between 0 and 1 ; complete uptake of $\mathrm{CO}$ is equal to 1 , and no uptake of $\mathrm{CO}$ is equal to 0.

Colon and ileum histopathology. Following euthanasia, entire colons were excised and fecal matter removed by gentle flushing with saline. Whole colons were fixed in formalin prior to paraffin embedding for slicing, staining (H\&E), and histological evaluation $(25,40)$. For quantitative PCR (qPCR) analysis, a longitudinal section of an entire length of colon was snap frozen and stored at $-80^{\circ} \mathrm{C}$ for subsequent RNA purification.

$R N A$ extraction, $c D N A$ synthesis, and $q P C R$ analysis. Freshly excised lung, colonic, and ileal tissues were snap frozen for subsequent storage at $-80^{\circ} \mathrm{C}$. RNA was prepared from colon and ileum using a previously described protocol (14-18). Tissue was thawed and homogenized ( $1 \mathrm{ml}$ of TRIzol, $4^{\circ} \mathrm{C}$; ThermoFisher Scientific) using a tissue-tearor homogenizer (Biospec). DNA was precipitated by chloroform addition, followed by centrifugation $(12,000 \mathrm{~g}, 15$ minutes), and the RNA-containing aqueous phase was collected. RNA was precipitated by addition of Isopropyl alchohol and pelleted prior to 2 washes with $75 \%$ ethanol. RNA pellets were dissolved in nuclease-free water, and RNA purity and quantity were assessed using a Nanodrop spectrophotometer. For cDNA synthesis, RNA (1,000 ng per sample) was preincubated with 1 unit of DNAse I (MilliporeSigma) (15 minutes, room temperature). Samples were heated $\left(65^{\circ} \mathrm{C}\right.$, 10 minutes) prior to the addition of random hexamer primers (Bioline) and dNTPs (Bioline), and further heating $\left(65^{\circ} \mathrm{C}, 5\right.$ minutes and $25^{\circ} \mathrm{C}, 10$ minutes). Reaction buffer, DTT, nuclease-free water, and Bioscript reverse transcriptase (Bioline) were added, and reverse transcription was completed $\left(10\right.$ minutes, $25^{\circ} \mathrm{C} ; 50$ minutes, $42^{\circ} \mathrm{C}$; and 15 minutes, $70^{\circ} \mathrm{C}$ ). qPCR analysis was performed in 384-well plates with SYBR-greenbased detection using a Viia 7 Real Time PCR system (ThermoFisher Scientific). For mRNA quantification from lung tissue, specific mRNA products were expressed as relative abundance compared with hypoxanthine-guanine phosphoribosyltransferase (HPRT), while - in colon and ileum tissue - mRNAs were expressed as abundance relative to $\beta$-actin expression.

$I H C$. For evaluation of tissue hypoxia in the GIT the pimonidazole-based probe Hypoxyprobe (Hypoxyprobe) was used as previously described $(24,31)$. Hypoxyprobe was administered (60 mg/kg, i.p.) 20 minutes prior to sacrifice. Hypoxyprobe signal was detected following the manufacturer's instructions. Briefly, 
paraffin embedded tissue slices were dewaxed and rehydrated prior to quenching of tissue peroxidases in $3 \%$ $\mathrm{H}_{2} \mathrm{O}_{2}$. Following antigen retrieval (standard citrate buffer, 20 minutes, $95^{\circ} \mathrm{C}$ ) slides were incubated (PBS + $0.05 \%$ Tween [PBS-T], 5 minutes, $2 \times$ ) before blocking (casein blocking buffer, 1 hour, room temperature). Slices were then incubated with primary antibody (1:50 in PBS-T, 40 minutes, room temperature). Secondary antibody was HRP-conjugated rabbit anti-mouse (MilliporeSigma, A9044, 1:200 in PBS-T). Hypoxyprobe related signals were visualized by incubating tissue slices in DAB chromogen (Dako, K3468) for equal lengths of time. Tissues were counterstained with hematoxylin, dehydrated, and mounted for imaging. For quantification, color deconvoluted images were assessed using ImageJ software for total hypoxyprobe DAB signal. Total hypoxyprobe signal in epithelial and mucosal layers was expressed as a percentage of epithelial and mucosal tissue that was hypoxyprobe positive. Additionally, the percentage of hypoxyprobe-positive epithelial and mucosal tissue was calculated separately and then expressed as a ratio of epithelial vs. mucosal levels of tissue hypoxia. Vasculature was visualized in a similar fashion using anti-mouse pan-endothelial cell antigen, clone MECA-32 primary antibody (Developmental Studies Hybridoma Bank, University of Iowa, Iowa City, Iowa, USA) and HRP-conjugated rabbit anti-rat HRP (Dako, P0162). The total number of individual MECA-32-positive events, percentage area of the epithelial and mucosal layers that was MECA32 positive, and the average size of MECA-32-labeled events was assessed.

TUNEL stain for cell death. For visualization and quantification of cell death in tissues, TUNEL assays were performed using the DeadEnd fluorometric TUNEL system (Roche Diagnostics) following the manufacturer's instructions. Formalin-fixed, paraffin-embedded tissue slices were dewaxed and dehydrated prior to further incubation (4\% paraformaldehyde, 15 minutes). Tissue was incubated with $20 \mu \mathrm{g} / \mathrm{ml}$ Proteinase $\mathrm{K}$, washed, and then incubated again in $4 \%$ paraformaldehyde. Samples were immersed in equilibration buffer (10 minutes) prior to addition of the rTdT enzyme/nucleotide labeling mix and incubation (60 minutes, $37^{\circ} \mathrm{C}$ ). The enzymatic reaction was terminated with $2 \times$ SSC buffer, nuclei were labeled with Hoechst $33342(5 \mu \mathrm{g} / \mathrm{ml})$, and tissue slices were mounted in FluorSave (MilliporeSigma) for imaging.

Intestinal permeability assay. Permeability of the colon and ileum was determined using a tissue sac method as previously described $(77,78)$. Briefly, following euthanasia, colonic and ileal tissues were excised and flushed with oxygenated Medium 199 (MilliporeSigma). One end of the colonic or ileal sac was carefully tied off. A second knot was tied loosely $2 \mathrm{~cm}$ away from the first knot. Each sac was then filled with $250 \mu \mathrm{l}$ of a solution of $1 \mathrm{mg} / \mathrm{ml}$ dextran-FITC (MilliporeSigma, FD4) dissolved in oxygenated Medium 199, and the second knot was tightened to create a sac. Excess tissue was removed, and the sacs were immersed in $15 \mathrm{ml}$ of Medium $199\left(37^{\circ} \mathrm{C}\right)$. Bathing Medium 199 (100 $\mu$ l) was sampled and replaced with fresh medium every 15 minutes. After 120 minutes, sacs were cut open, and contents were sampled. FD4 concentration in media samples was measured using a FLUOstar microplate reader (BMG Labtech). Velocity of escape of FD4 across the intestinal layer was calculated to yield the apparent permeability of the colon and ileum to FD4 $\left(P_{a p p}\right)$ using the equation $P_{a p p}\left(\mathrm{~cm} \mathrm{~s}^{-1}\right)=(d Q / d t) /(A \mathrm{Co})$, where $d Q / d t$ is the transport rate (mols ${ }^{-1}$ of FD4 $), A$ is the surface area of the sac $\left(\mathrm{cm}^{2}\right)$, and Co is the initial concentration of FD4 injected into the sac $\left(\mathrm{mol} \mathrm{ml} l^{-1}\right)$.

Patient recruitment and human clinical samples. Twelve patients with $\mathrm{CD}$ were recruited at Royal Brisbane and Women's Hospital with a validated protocol-driven approach (79), and diagnosis was confirmed by histopathology. Twelve asymptomatic individuals undergoing colonoscopy for screening purposes, with no history of gastrointestinal inflammatory disease, were recruited as control subjects. Patients were age and sex matched between groups. Patient ages ranged from 24-72 years of age (Mean age, 47 years). Smoking status was recorded at time of recruitment, with ex-smokers defined as those individuals who had abstained from smoking for at least 6 months prior to recruitment. Serum was collected from whole blood and stored at $-80^{\circ} \mathrm{C}$ until analysis. Serum VEGF levels were measured by ELISA using R\&D Systems Human VEGF DuoSet ELISA Kit, as per the manufacturer's instructions. For clinical biopsy samples of terminal ileum, tissue was formalin fixed and embedded in paraffin. Vasculature was visualized through detection of CD34 (Leica, NCL-L-END, 1:200) with a Bond Polymer Refine detection system (Leica).

Statistics. Statistical analysis was performed using Prism 6 software (GraphPad). The data were analyzed by 1-way ANOVA with post hoc Tukey's test or by Student's unpaired 2-tailed $t$ test as appropriate. $P<0.05$ was considered statistically significant.

Study approval. Informed consent was obtained from all patients, and the Royal Brisbane and Women's Hospital human research ethics committee approved the clinical study protocol. All mouse experiments were approved by the University of Newcastle animal ethics committee. 


\section{Author contributions}

MF designed research studies, conducted experiments, analyzed data, and wrote the manuscript. BJG and SM acquired data. BJ, RYK, SLG, and AGJ conducted experiments. BGO designed research studies. GRS designed research studies and collected clinical data. NP designed research studies and edited the manuscript. NJT designed research studies, analyzed data, and edited the manuscript. MMW designed research studies, analyzed data, and edited the manuscript. SK designed research studies, analyzed data, and edited the manuscript. PMH designed research studies, analyzed data, and edited the manuscript.

\section{Acknowledgments}

The authors would like to thank professors Mark Inman (McMaster University) and Wayne Mitzner (Johns Hopkins University) for their advice on $\mathrm{DF}_{\mathrm{CO}}$ testing, and Kristy Wheeldon and Mr Matthew Bowman for their expert technical assistance. PMH is supported by a National Health and Medical Research Council (NHMRC) Principal Research Fellowship and a Brawn Fellowship from the Faculty of Health and Medicine at The University of Newcastle. MF is currently supported by an NHMRC Research Fellowship with in the Centre of Excellence in Severe Asthma at The University of Newcastle. SK is currently supported by a Brawn Fellowship from the University of Newcastle and a Cancer Institute NSW Career Development Fellowship. This work was supported by NHMRC grants to PMH, NJT, BGO, and SK.

Address correspondence to: Philip M. Hansbro, HMRI, Lot 1 Kookaburra Circuit, New Lambton Heights, 2305, NSW, Australia. Phone: 61.0.240420187; Email: Philip.Hansbro@newcastle.edu.au.

1. Lozano R, et al. Global and regional mortality from 235 causes of death for 20 age groups in 1990 and 2010: a systematic analysis for the Global Burden of Disease Study 2010. Lancet. 2012;380(9859):2095-2128.

2. Fricker M, Deane A, Hansbro PM. Animal models of chronic obstructive pulmonary disease. Expert Opin Drug Discov. 2014;9(6):629-645.

3. Chambers DC, Gellatly SL, Hugenholtz P, Hansbro PM. JTD special edition 'Hot Topics in COPD'-The microbiome in COPD. J Thorac Dis. 2014;6(11):1525-1531.

4. Ventham NT, Kennedy NA, Nimmo ER, Satsangi J. Beyond gene discovery in inflammatory bowel disease: the emerging role of epigenetics. Gastroenterology. 2013;145(2):293-308.

5. Kaplan GG. The global burden of IBD: from 2015 to 2025. Nat Rev Gastroenterol Hepatol. 2015;12(12):720-727.

6. Keely S, Talley NJ, Hansbro PM. Pulmonary-intestinal cross-talk in mucosal inflammatory disease. Mucosal Immunol. 2012;5(1):7-18.

7. Ananthakrishnan AN. Epidemiology and risk factors for IBD. Nat Rev Gastroenterol Hepatol. 2015;12(4):205-217.

8. Ekbom A, Brandt L, Granath F, Löfdahl CG, Egesten A. Increased risk of both ulcerative colitis and Crohn's disease in a population suffering from COPD. Lung. 2008;186(3):167-172.

9. Birrenbach T, Böcker U. Inflammatory bowel disease and smoking: a review of epidemiology, pathophysiology, and therapeutic implications. Inflamm Bowel Dis. 2004;10(6):848-859.

10. To N, Gracie DJ, Ford AC. Systematic review with meta-analysis: the adverse effects of tobacco smoking on the natural history of Crohn's disease. Aliment Pharmacol Ther. 2016;43(5):549-561.

11. Budden KF, et al. Emerging pathogenic links between microbiota and the gut-lung axis. Nat Rev Microbiol. 2017;15(1):55-63

12. Lakatos PL, Szamosi T, Lakatos L. Smoking in inflammatory bowel diseases: good, bad or ugly? World J Gastroenterol. 2007;13(46):6134-6139.

13. Rutten EPA, Lenaerts K, Buurman WA, Wouters EFM. Disturbed intestinal integrity in patients with COPD: effects of activities of daily living. Chest. 2014;145(2):245-252.

14. Beckett EL, et al. A new short-term mouse model of chronic obstructive pulmonary disease identifies a role for mast cell tryptase in pathogenesis. J Allergy Clin Immunol. 2013;131(3):752-762.

15. Hansbro PM, et al. Importance of mast cell Prss31/transmembrane tryptase/tryptase- $\gamma$ in lung function and experimental chronic obstructive pulmonary disease and colitis. J Biol Chem. 2014;289(26):18214-18227.

16. Franklin BS, et al. The adaptor ASC has extracellular and 'prionoid' activities that propagate inflammation. Nat Immunol. 2014;15(8):727-737.

17. Hsu AC, et al. Targeting PI3K-p110 $\alpha$ Suppresses Influenza Virus Infection in Chronic Obstructive Pulmonary Disease. Am J Respir Crit Care Med. 2015;191(9):1012-1023.

18. Tay HL, et al. Antagonism of miR-328 increases the antimicrobial function of macrophages and neutrophils and rapid clearance of non-typeable Haemophilus influenzae (NTHi) from infected lung. PLoS Pathog. 2015;11(4):e1004549.

19. Liu G, et al. Fibulin-1 regulates the pathogenesis of tissue remodeling in respiratory diseases. JCI Insight. 2016;1(9):e86380.

20. Haw TJ, et al. A pathogenic role for tumor necrosis factor-related apoptosis-inducing ligand in chronic obstructive pulmonary disease. Mucosal Immunol. 2016;9(4):859-872.

21. Limjunyawong N, Mitzner W, Horton MR. A mouse model of chronic idiopathic pulmonary fibrosis. Physiol Rep. 2014;2(2):e00249.

22. Harvey BG, et al. Risk of COPD with obstruction in active smokers with normal spirometry and reduced diffusion capacity Eur Respir J. 2015;46(6):1589-1597. 
23. Makita $\mathrm{H}$, et al. Characterisation of phenotypes based on severity of emphysema in chronic obstructive pulmonary disease. Thorax. 2007;62(11):932-937.

24. Robinson A, Keely S, Karhausen J, Gerich ME, Furuta GT, Colgan SP. Mucosal protection by hypoxia-inducible factor prolyl hydroxylase inhibition. Gastroenterology. 2008;134(1):145-155.

25. Marks E, et al. Oral delivery of prolyl hydroxylase inhibitor: AKB-4924 promotes localized mucosal healing in a mouse model of colitis. Inflamm Bowel Dis. 2015;21(2):267-275.

26. Neurath MF. Cytokines in inflammatory bowel disease. Nat Rev Immunol. 2014;14(5):329-342.

27. Koutroubakis IE, Tsiolakidou G, Karmiris K, Kouroumalis EA. Role of angiogenesis in inflammatory bowel disease. Inflamm Bowel Dis. 2006;12(6):515-523.

28. Suski MD, Zabel D, Levin V, Scheuenstuhl H, Hunt TK. Effect of hypoxic hypoxia on transmural gut and subcutaneous tissue oxygen tension. Adv Exp Med Biol. 1997;411:319-322.

29. Biddlestone J, Bandarra D, Rocha S. The role of hypoxia in inflammatory disease (review). Int J Mol Med. 2015;35(4):859-869.

30. Raleigh JA, Chou SC, Arteel GE, Horsman MR. Comparisons among pimonidazole binding, oxygen electrode measurements, and radiation response in C3H mouse tumors. Radiat Res. 1999;151(5):580-589.

31. Harris NR, et al. Relationship between inflammation and tissue hypoxia in a mouse model of chronic colitis. Inflamm Bowel Dis 2011;17(3):742-746

32. Goggins BJ, Chaney C, Radford-Smith GL, Horvat JC, Keely S. Hypoxia and Integrin-Mediated Epithelial Restitution during Mucosal Inflammation. Front Immunol. 2013;4:272

33. Hallmann R, Mayer DN, Berg EL, Broermann R, Butcher EC. Novel mouse endothelial cell surface marker is suppressed during differentiation of the blood brain barrier. Dev Dyn. 1995;202(4):325-332.

34. Kimura $\mathrm{H}$, et al. Hypoxia response element of the human vascular endothelial growth factor gene mediates transcriptional regulation by nitric oxide: control of hypoxia-inducible factor-1 activity by nitric oxide. Blood. 2000;95(1):189-197.

35. Hindryckx P, et al. Hydroxylase inhibition abrogates TNF-alpha-induced intestinal epithelial damage by hypoxia-inducible factor-1-dependent repression of FADD. J Immunol. 2010;185(10):6306-6316.

36. Negroni A, Cucchiara S, Stronati L. Apoptosis, Necrosis, and Necroptosis in the Gut and Intestinal Homeostasis. Mediators Inflamm. 2015;2015:250762.

37. Lindberg E, Järnerot G, Huitfeldt B. Smoking in Crohn's disease: effect on localisation and clinical course. Gut. 1992;33(6):779-782.

38. Bustamante M, et al. Relationship between smoking and colonic involvement in inflammatory bowel disease. Rev Esp Enferm Dig. 1998;90(12):833-840.

39. Cosnes J, Beaugerie L, Carbonnel F, Gendre JP. Smoking cessation and the course of Crohn's disease: an intervention study. Gastroenterology. 2001;120(5):1093-1099.

40. Keely S, et al. Contribution of epithelial innate immunity to systemic protection afforded by prolyl hydroxylase inhibition in murine colitis. Mucosal Immunol. 2014;7(1):114-123.

41. Park HY, et al. Serum vascular endothelial growth factor and angiopoietin-2 are associated with the severity of systemic inflammation rather than the presence of hemoptysis in patients with inflammatory lung disease. Yonsei Med J. 2012;53(2):369-376.

42. Dev A, Patel K, Conrad A, Blatt LM, McHutchison JG. Relationship of smoking and fibrosis in patients with chronic hepatitis C. Clin Gastroenterol Hepatol. 2006;4(6):797-801.

43. Matsuyama W, et al. Serum levels of vascular endothelial growth factor dependent on the stage progression of lung cancer. Chest. 2000;118(4):948-951.

44. Griga T, Tromm A, Spranger J, May B. Increased serum levels of vascular endothelial growth factor in patients with inflammatory bowel disease. Scand J Gastroenterol. 1998;33(5):504-508.

45. Allais L, et al. Chronic cigarette smoke exposure induces microbial and inflammatory shifts and mucin changes in the murine gut. Environ Microbiol. 2016;18(5):1352-1363.

46. Calverley PM, Leggett RJ, McElderry L, Flenley DC. Cigarette smoking and secondary polycythemia in hypoxic cor pulmonale. Am Rev Respir Dis. 1982;125(5):507-510.

47. El-Zayadi AR, Selim O, Hamdy H, El-Tawil A, Moustafa H. Heavy cigarette smoking induces hypoxic polycythemia (erythrocytosis) and hyperuricemia in chronic hepatitis $\mathrm{C}$ patients with reversal of clinical symptoms and laboratory parameters with therapeutic phlebotomy. Am J Gastroenterol. 2002;97(5):1264-1265.

48. Hasselbalch HC. Smoking as a contributing factor for development of polycythemia vera related neoplasms [published online ahead of print September 9, 2015]. Leuk Res. https://doi.org/0.1016/j.leukres.2015.09.002.

49. Srivastava ED, Russell MA, Feyerabend C, Rhodes J. Effect of ulcerative colitis and smoking on rectal blood flow. Gut. 1990;31(9):1021-1024

50. Di Sabatino A, et al. Serum bFGF and VEGF correlate respectively with bowel wall thickness and intramural blood flow in Crohn's disease. Inflamm Bowel Dis. 2004;10(5):573-577.

51. Deng X, et al. New cell therapy using bone marrow-derived stem cells/endothelial progenitor cells to accelerate neovascularization in healing of experimental ulcerative colitis. Curr Pharm Des. 2011;17(16):1643-1651.

52. Cromer WE, Mathis JM, Granger DN, Chaitanya GV, Alexander JS. Role of the endothelium in inflammatory bowel diseases. World J Gastroenterol. 2011;17(5):578-593.

53. Loboda A, Jozkowicz A, Dulak J. HIF-1 and HIF-2 transcription factors--similar but not identical. Mol Cells. 2010;29(5):435-442.

54. Zhao J, Du F, Shen G, Zheng F, Xu B. The role of hypoxia-inducible factor-2 in digestive system cancers. Cell Death Dis. 2015;6:e1600.

55. Botteri E, Iodice S, Bagnardi V, Raimondi S, Lowenfels AB, Maisonneuve P. Smoking and colorectal cancer: a meta-analysis. JAMA. 2008;300(23):2765-2778.

56. Shepherd AP, Kiel JW. A model of countercurrent shunting of oxygen in the intestinal villus. Am J Physiol. 1992;262(4 Pt 2):H1136-H1142.

57. Scharff JR, Longo WE, Vartanian SM, Jacobs DL, Bahadursingh AN, Kaminski DL. Ischemic colitis: spectrum of disease and outcome. Surgery. 2003;134(4):624-629.

58. Danese S. VEGF in inflammatory bowel disease: a master regulator of mucosal immune-driven angiogenesis. Dig Liver Dis. 
2008;40(8):680-683.

59. Scaldaferri F, et al. VEGF-A links angiogenesis and inflammation in inflammatory bowel disease pathogenesis. Gastroenterology. 2009;136(2):585-95.e5.

60. Ikeda $\mathrm{H}$, et al. Apoptosis is a major mode of cell death caused by ischaemia and ischaemia/reperfusion injury to the rat intestinal epithelium. Gut. 1998;42(4):530-537.

61. Takahashi N, et al. RIPK1 ensures intestinal homeostasis by protecting the epithelium against apoptosis. Nature. 2014;513(7516):95-99.

62. Lau E, Carvalho D, Freitas P. Gut Microbiota: Association with NAFLD and Metabolic Disturbances. Biomed Res Int. 2015;2015:979515.

63. Liu Y, et al. Active smoking, passive smoking, and risk of nonalcoholic fatty liver disease (NAFLD): a population-based study in China. J Epidemiol. 2013;23(2):115-121.

64. Wong VW, et al. Bacterial endotoxin and non-alcoholic fatty liver disease in the general population: a prospective cohort study Aliment Pharmacol Ther. 2015;42(6):731-740.

65. Fortunato F, et al. Pancreatic response to endotoxin after chronic alcohol exposure: switch from apoptosis to necrosis? $A m J$ Physiol Gastrointest Liver Physiol. 2006;290(2):G232-G241.

66. Maisonneuve P, et al. Cigarette smoking accelerates progression of alcoholic chronic pancreatitis. Gut. 2005;54(4):510-514.

67. Andriulli A, et al. Smoking as a cofactor for causation of chronic pancreatitis: a meta-analysis. Pancreas. 2010;39(8):1205-1210.

68. He G, Shankar RA, Chzhan M, Samouilov A, Kuppusamy P, Zweier JL. Noninvasive measurement of anatomic structure and intraluminal oxygenation in the gastrointestinal tract of living mice with spatial and spectral EPR imaging. Proc Natl Acad Sci USA. 1999;96(8):4586-4591.

69. Albenberg L, et al. Correlation between intraluminal oxygen gradient and radial partitioning of intestinal microbiota. Gastroenterology. 2014;147(5):1055-63.e8

70. Fujita N, Chiba K, Shapiro IM, Risbud MV. HIF-1 $\alpha$ and HIF-2 $\alpha$ degradation is differentially regulated in nucleus pulposus cells of the intervertebral disc. J Bone Miner Res. 2012;27(2):401-412.

71. Starkey MR, et al. Murine models of infectious exacerbations of airway inflammation. Curr Opin Pharmacol. 2013;13(3):337-344.

72. Starkey MR, et al. Chlamydia muridarum lung infection in infants alters hematopoietic cells to promote allergic airway disease in mice. PLoS One. 2012;7(8):e42588.

73. Foster PS, et al. The emerging role of microRNAs in regulating immune and inflammatory responses in the lung. Immunol Rev. 2013;253(1):198-215

74. Cummins EP, et al. The hydroxylase inhibitor dimethyloxalylglycine is protective in a murine model of colitis. Gastroenterology. 2008;134(1):156-165.

75. Ruifrok AC, Johnston DA. Quantification of histochemical staining by color deconvolution. Anal Quant Cytol Histol. 2001;23(4):291-299.

76. Horvat JC, et al. Early-life chlamydial lung infection enhances allergic airways disease through age-dependent differences in immunopathology. J Allergy Clin Immunol. 2010;125(3):617-625.

77. Keely S, et al. In vitro and ex vivo intestinal tissue models to measure mucoadhesion of poly (methacrylate) and N-trimethylated chitosan polymers. Pharm Res. 2005;22(1):38-49.

78. Mateer SW, Cardona J, Marks E, Goggin BJ, Hua S, Keely S. Ex Vivo Intestinal Sacs to Assess Mucosal Permeability in Models of Gastrointestinal Disease. J Vis Exp. 2016;(108):e53250.

79. Simms LA, Doecke JD, Walsh MD, Huang N, Fowler EV, Radford-Smith GL. Reduced alpha-defensin expression is associated with inflammation and not NOD2 mutation status in ileal Crohn's disease. Gut. 2008;57(7):903-910. 